\title{
A Self-Tuning zSlices based General Type-2 Fuzzy PI Controller
}

\author{
Tufan Kumbasar, Member, IEEE and Hani Hagras, Fellow, IEEE
}

\begin{abstract}
The Interval Type-2 Fuzzy PI controller (IT2-FPI) might be able to handle high levels of uncertainties to produce a satisfactory control performance which could be potentially due to the robust performance as a result of the smoother control surface around the steady state [1]. However, the transient state and disturbance rejection performance of the IT2-FPI may degrade in comparison to the Type-1 Fuzzy PI (T1-FPI) counterpart [1]. This drawback can be resolved via general type2 fuzzy PI controllers which can provide a trade-off between the robust control performance of the IT2-FPI and the acceptable transient and disturbance rejection performance of the type-1 PI controllers. In this paper, we will present a zSlices based general Type-2 Fuzzy PI controller (zT2-FPI) where the Secondary Membership Functions (SMFs) of the antecedent general type-2 fuzzy sets are adjusted in an on-line manner. We will examine the effect of the SMF on the closed system control performance to investigate their induced performance improvements. The paper will focus on the case followed in conventional or self-tuning fuzzy controller design strategies where the aim is to decrease the integral action sufficiently around the steady state to have robust system performance against noises and parameter variations. The zSlices approach will give the opportunity to construct the zT2-FPI controller as a collection of IT2-FPI and T1-FPI controllers. We will present a new way to design a zT2-FPI controller based on a single tuning parameter where the features of T1-FPI (speed) and IT2-FPI (robustness) are combined without increasing the computational complexity much when compared to the IT2-FPI structure. This will allow the proposed zT2-FPI controller to achieve the desired transient state response and provide an efficient disturbance rejection and robust control performance. We will present several simulation studies on benchmark systems in addition to real-world experiments which were performed using the PIONEER 3-DX mobile robot that will act as a platform to evaluate the proposed systems. The results will show that the control performance of the self-tuning zT2-FPI control structure enhances both the transient state and disturbance rejection performances when compared to the type-1 and IT2-FPI counterparts. In addition, the self-tuning zT2-FPI is more robust to disturbances, noise and uncertainties when compared to the type-1 and interval type- 2 fuzzy counterparts.
\end{abstract}

Index Terms-General Type-2 Fuzzy Logic Systems, zSlices based General Type-2 Fuzzy Logic Systems, Interval Type-2 Fuzzy Logic Systems

This research is supported by the project (113E206) of Scientific and Technological Research Council of Turkey (TUBITAK). All of these supports are appreciated.

T. Kumbasar is with the Control and Automation Engineering Department, Faculty of Electrical and Electronics Engineering, Istanbul Technical University, Istanbul, TR-34469, Turkey (e-mail: kumbasart@itu.edu.tr).

H. Hagras is with the Computational Intelligence Centre, School of Computer Science and Electronic Engineering, University of Essex, Colchester CO4 3SQ, United Kingdom (e-mail: hani@essex.ac.uk).)

\section{INTRODUCTION}

$\mathrm{R}$ ecently, Interval Type-2 Fuzzy Logic Controllers (IT2FLCs) attracted significant research interest especially in controlling nonlinear and uncertain systems. The IT2-FLCs demonstrated control performance improvements which could be attributed to the additional degree of freedom provided by the Footprint of Uncertainty (FOU) present in the antecedent interval type-2 fuzzy Membership Functions (MFs) [2-6]. The internal structure of the IT2-FLC is similar to its type-1 counterpart. However, the major differences are that IT2-FLCs employ Interval Type-2 Fuzzy Sets (IT2-FSs) (rather than type-1 fuzzy sets) and the IT2-FLCs process IT2-FSs and thus the IT2-FLC has an extra type-reduction process [7-9]. Nevertheless, the systematic design of IT2-FLCs is still a challenging problem due to the main difficulty in determining the parameters of the Fuzzy Sets (FSs) and the rulebase [10]. Recently, several studies have employed various techniques for the design of IT2-FLCs including genetic algorithms [4], [11], particle swarm [12] and ant colony optimization [13].

In the IT2-FLC literature, several studies have been presented to analyze and examine the behavior of the interval type-2 fuzzy PI and PD controllers [1], [14-16]. Wu and Tan [1] showed that the Interval Type-2 Fuzzy PI controllers (IT2FPI) are generally more robust than their type-1 counterparts by examining their behavior around the steady state. It has been also reported that the IT2-FPI controller behaves like a variable gain PI controller and has a smoother control surface around the steady state in comparison with its type- 1 and conventional controllers counterparts [1]. Thus, tuning the FOUs of the IT2-FSs of the IT2-FPI might potentially result in more robust controllers since a smooth control surface might be generated [1]. However, the IT2-FPI transient state and disturbance rejection performance may degrade in comparison with its type-1 and conventional counterparts [1], [14]. Although a smooth control surface is probably a common objective in industrial practice, the problem is that the resulting disturbance response may be unacceptable (too slow) since disturbances occurring around the steady state might cause a smaller control output change [17]. This problem is usually solved by a trade-off between control performance and robustness [18].

General type-2 fuzzy PI controllers can provide an acceptable trade-off between the robust control performance of the IT2-FPI and the acceptable transient and disturbance 
rejection performance of the type-1 PI controllers. Thus the general type-2 fuzzy PI controller might be able to enhance both the transient state and disturbance rejection performances while preserving the robustness of the type-2 fuzzy controller. In this context, we will examine and present the effect of the shape and size of the Secondary MFs (SMFs) of the type-2 fuzzy controller on the control performance.

General Type-2 Fuzzy Sets (T2-FSs) give the opportunity to define the uncertainty in the third dimension (the shape of the SMFs). Fig. 1a shows the front projection on the $x-u$ plane of a given general T2-FS $(\tilde{F})$ while Fig.1b shows the SMF of the T2-FS at $x$ '. T2-FSs are relatively more complex than the IT2FSs due to the need to determine the shape and parameters of the third dimension. Several forms of representations of general T2-FSs have been developed to enable the use of general type-2 Fuzzy Logic Systems (FLSs) in real-world applications [19-23]. In this paper, we will employ the zSlices based general Type-2 Fuzzy Sets (zT2-FSs) and zSlices based general Type-2 Fuzzy Logic Systems (zT2-FLSs) which were proposed by Wagner and Hagras [23]. The zSlices theory gives the opportunity to calculate the crisp output of the zT2FLS by using the fact that zT2-FLS can be implemented by constructing a series of interval type-2 fuzzy logic systems which are associated with different zLevels [23]. The zT2-FLS approach has been implemented successfully in real-world applications [23], [24].

In this paper, we will present a novel zSlices based general Type-2 Fuzzy PI (zT2-FPI) controller where the SMFs are adjusted in an on-line manner through a single tuning parameter. The proposed zT2-FPI structure and tuning mechanism will give the opportunity to improve the transient state response while enhancing the disturbance rejection performance in addition to improving the system robustness. We will first present the structure of the proposed novel zT2FPI controller and show that the SMF of the general T2-FSs can easily be tuned by a single tuning parameter. In order to analyze the behavior of the zT2-FPI controller around the steady state, we will first provide the mathematical background of an IT2-FPI controller since the zT2-FPI controller structure can be seen as a collection of IT2-FPIs which are associated with different zLevels (we will follow the same analysis strategy presented in [1], [14] while using triangular IT2-FSs instead of trapezoidal IT2-FSs). Afterwards, we will analyze and provide theoretical explanations of why the zT2-FPIs are able to eliminate oscillations and be potentially more robust against parameter variations when compared to Type-1 Fuzzy PI (T1-FPI) controllers. We will investigate the effect of the SMFs in two parts which are the size (relative to the FOU) and the shape of the SMFs. Based on the observations; we will present two heuristic tuning mechanisms based on the feedback error. In the first proposed self-tuning zT2-FPI structure, where the SMFs are interval sets (thus having a self-tuning IT2-FPI structure), the FOU size is tuned in an online manner. In the second control structure, the shape of the SMF with a fixed FOU size is online tuned. Then, we will present an optimization based design strategy for the design of the proposed zT2-FPI control structures.

We will present several simulation studies to validate the proposed approaches, where we will present first simulation studies where the proposed Error based self-Tuning zT2-FPI (EzT2-FPI) control structures are compared with an Optimized Type-1 Fuzzy PI (OT1-FPI) and Interval Type-2 Fuzzy PI (OIT2-FPI) control structures. The comparative simulation results will show that the control performance of the both EzT2-FPI structures improved both the transient state and disturbance rejection performances of the OT1-FPI and OIT2FPI. However, it will be illustrated that the SMF Shape Tuning strategy based zT2-FPI (ST-zT2-FPI) control system is more robust against nonlinear dynamics, parameter variations, disturbances and noise in comparison to the FOU size Tuning strategy based zT2-FPI (FT-zT2-FPI) (which is in fact a selftuning IT2-FPI controller). Moreover, we will show that the proposed zT2-FPI controller has a reasonable computational cost which makes it feasible for real-time control applications since it is constructed based on zSlices theory and tuned by a single parameter. We will also evaluate the various fuzzy PI control systems on the real-time path tracking problem of the real-world PIONEER 3-DX mobile robot which inherits large amounts of nonlinearities and uncertainties caused by the internal dynamics and/or feedback sensors of the controlled system. The comparative real-time control studies have shown that the overall control performance of the EzT2-FPI is better in terms of transient state and disturbance rejection and it is also more robust against nonlinear dynamics, parameter variations and disturbances when compared to its optimized type-1 and interval type-2 fuzzy controller counterparts.

Section II will briefly present an overview of T1-FPI and IT2-FPI controllers. Section III will present the structure of the proposed the zT2-FPI controller. Section IV will present the effect of the SMFs on the controller performance. Section $\mathrm{V}$ will present the proposed error based self-tuning mechanisms. Section VI will briefly present the design methodology of the proposed zT2-FPI structure. Section VII will present the simulation studies for linear and nonlinear systems while the real world experiments results are presented in Section VIII. Section IX will present the conclusions and future work.

\section{BRIEF OVERVIEW ABOUT TYPE-1 AND INTERVAL TYPE-2 FUZZY PI CONTROLLERS}

In this section, we will present brief information about the T1-FPI and IT2-FPI controllers which are used in the comparison studies (and to construct the proposed zT2-FPI controller).

A linear PI control law can be implemented as:

$$
\dot{u}=K_{P} \dot{e}+K_{I} e
$$

where $\dot{u}$ is the change of the control signal, $e$ is the feedback error, $\dot{e}$ is the change of the feedback error, and $K_{P}$ and $K_{I}$ are the proportional and integral gains, respectively. Moreover, the controller gain and integral time constant of the PI controller are defined as $K_{c}=K_{P}$ and $T_{i}=K_{I} / K_{P}$, respectively. 
It has been shown that a rapid generation of fuzzy rules based on the existing linear PI controller can be achieved [1], [25]. Thus, a T1-FPI with the rulebase whose rule is represented as follows:

$$
\begin{gathered}
R^{i j}: \mathrm{IF} \dot{e} \text { is } \dot{E}_{i} \text { and } e \text { is } E_{j} \text { Then } \dot{u} \text { is } \dot{u}_{i j} \\
i=-N, \ldots N ; j=-M, \ldots M
\end{gathered}
$$

has an identical output to the PI control law if [1], [25]:

(i) $50 \%$ overlapping T1-FSs are used for input MFs $\left(\dot{E}_{i}, E_{j}\right)$, i.e, $\sum_{i=1}^{2 M} \mu_{\dot{E}_{i}}=1$ and $\sum_{j=1}^{2 N} \mu_{E_{j}}=1$ where $2 M$ and $2 N$ are the numbers of the MFs for the inputs $\dot{e}$ and $e$, respectively. The illustration of such MFs is shown as the bold lines in Fig. 2 where $c_{\dot{e} i}, c_{e j}$ are the cores of the MFs.

(ii) The consequents of the rules are crisp numbers defined as follows:

$$
\dot{u}_{i j}=K_{P} c_{\dot{e} i}+K_{I} c_{e j}
$$

The IT2-FSs used in an IT2-FPI can be simply constructed by extending the T1-FSs of a T1-FPI, as shown in Fig. 2. The rulebase for an IT2-FPI is defined as follows:

$$
\begin{gathered}
R^{i j}: \mathrm{IF} \dot{e} \text { is } \tilde{E}_{i} \text { and } e \text { is } \tilde{E}_{j} \text { Then } \dot{u} \text { is } \dot{u}_{i j} \\
i=-N, \ldots N ; j=-M, \ldots M
\end{gathered}
$$

where $\tilde{E}_{i}$ and $\widetilde{E}_{j}$ are antecedent IT2-FSs obtained by extending the T1-FSs $\left(\dot{E}_{i}\right.$ and $\left.E_{j}\right)$, and $\dot{u}_{i j}$ is defined in Equation (3). The IT2-FSs are described in terms of upper MFs $\left(\bar{\mu}_{\tilde{E}_{i}}, \bar{\mu}_{\tilde{E}_{j}}\right)$ and lower MFs $\left(\underline{\mu}_{\tilde{E}_{i}}{ }^{\prime} \underline{\tilde{E}}_{\tilde{E}_{j}}\right)$ which are defined with their cores $\left(c_{\dot{e} i}, c_{e j}\right)$ and the height of their MFs $\left(\alpha_{\dot{e} i}, \alpha_{e j}\right)$ as illustrated in Fig. 2. The IT2-FSs have the following properties.

$$
\begin{array}{lll}
\text { i) } & \sum_{i=1}^{2 M} \bar{\mu}_{\tilde{E}_{i}}=1 & , \sum_{i=1}^{2 N} \bar{\mu}_{\tilde{E}_{j}}=1 \\
\text { ii) } & \underline{\mu}_{\tilde{E}_{i}}=\bar{\mu}_{\tilde{E}_{i}} \alpha_{\dot{e} i} & , \underline{\mu}_{\tilde{E}_{j}}=\bar{\mu}_{\tilde{E}_{j}} \alpha_{e j} \\
\text { iii) } & c_{\dot{e} i}=-c_{\dot{e}-i} & , c_{e j}=-c_{e-j} \\
\text { iv) } & \alpha_{\dot{e} j}=\alpha_{\dot{e}-j} & , \alpha_{e i}=\alpha_{e-i}
\end{array}
$$

The implemented IT2-FPI uses the center of sets Type Reduction (TR) method [7]. Thus, the defuzzified output $\left(\dot{u}_{I T 2}\right)$ of an IT2-FPI can be calculated as:

$$
\dot{u}_{I T 2}=\left(\dot{u}_{l}+\dot{u}_{r}\right) / 2
$$

where $\dot{u}_{l}$ and $\dot{u}_{r}$ are the left and right end points respectively of the type reduced set. The typed reduced set can be calculated by using the iterative Karnik and Mendel (KM) TR procedure [26].

\section{The ZSLices BAsed General TyPe-2 FuZzy PI CONTROLLER STRUCTURE}

In this paper, we will employ the zSlices theory to design a novel zT2-FPI controller structure. We will firstly present information about the zSlices theory which will facilitate the design of the zT2-FPI since it can be seen as a collection of IT2-FPI controllers. We will present a simple design method for the zT2-FPI controllers based on one tuning parameter which will allow facilitating the design of the SMFs of the T2FSs. Since the output of the zT2-FPI is an aggregation of the outputs of several IT2-FPIs each associated with a specific zLevel, we will first derive the closed form formulation of an
IT2-FPI around the steady state. Then, we will combine the IT2-FPIs each associated with a specific zLevel and derive the output of the zT2-FPI around the steady state.

\section{A. Brief Information about zSlices based General Type-2 Fuzzy Sets and Systems}

The concept of zT2-FLSs was introduced by Wagner and Hagras [23]. The internal structure of the zT2-FLS is very similar to its interval type-2 counterpart, i.e. it is composed of a Fuzzifier, an Inference Engine and Rule Base as well as a Type-Reducer and Defuzzifier. However, the major difference is that zT2-FLSs employ and process zT2-FSs. A zSlice $\tilde{Z}_{q}$ is formed by slicing a T2-FS in the third dimension $z$ at level $z_{q}$ [21]. A zSlice $\tilde{Z}_{q}$ is defined as:

$$
\tilde{Z}_{q}=\int_{x \in X} \int_{u_{q} \in J_{q_{x}}} z_{q} /\left(x, u_{q}\right), J_{q_{x}} \in\left[l_{q}, r_{q}\right]
$$

Hence, at each $x$ value, z-Slicing creates an interval set with the height $z_{q}$ in the domain $J_{q_{x}}$ which ranges from $l_{q}$ to $r_{q}$, $0 \leq q \leq Q, Q$ is the number of zSlices (excluding $\tilde{Z}_{0}$ ) and $z_{q}=1 / Q . \tilde{Z}_{0}$ is a special case since its height is $z=0$ and therefore can be neglected [21]. The concept of the z-Slicing is given in Fig. $1 \mathrm{~b}$ for $Q=3$ zSlices. Thus, a T2-FS $(\tilde{F})$ can be seen equivalent to the collection of $Q$ zSlices as follows:

$$
\tilde{F}=\sum_{q=1}^{Q} \tilde{Z}_{q}
$$

This gives the opportunity to calculate the crisp output of the zT2-FLS by using the fact that zT2-FLS can be implemented by constructing a series of IT2-FLSs associated with various zLevels [23]. Thus, the outputs of all IT2-FLSs (within a zT2FLS) are fused to obtain the zT2-FLS output $\left(y_{c}\right)$ of the as follows:

$y_{c}=\frac{z_{1} \frac{\left(y_{l_{1}}+y_{r_{1}}\right)}{2}+z_{2} \frac{\left(y_{l_{2}}+y_{r_{2}}\right)}{2} \cdots+z_{Q} \frac{\left(y_{l_{Q}}+y_{r_{Q}}\right)}{2}}{z_{1}+z_{2}+\cdots+z_{Q}}$

where $\left(y_{l_{q}}, y_{r_{q}}\right)$ is the type reduced set for each zSlice related IT2-FLS which is obtained by the center of sets TR method [24]. At the zSlice level $z_{Q}, y_{l_{Q}}=y_{r_{Q}}$, then the IT2-FLS for the zSlice level $z_{Q}$ will reduce to a T1-FLS. A detailed comprehensive analysis on the zT2-FLS can be found in [23].

We would like to point out that the employed zT2-FLS in this paper can also be seen as a quasi T2-FLS which represents the T2-FSs via an alpha-level representation [20]. In [27], it has been proven that there is an equivalence between the alpha-level and zSlices representations of T2-FSs. Thus, the proposed zSlices representation based general type-2 fuzzy controller structure (which will be introduced in the following section) can also be constructed with the equivalent representation of alpha-level representation.

\section{B. The General Structure and the Components of the zT2-FPI Controller}

In this subsection, we will present the general structure and the components of the zT2-FPI controller. As has been explained in the previous subsection, the output of the zT2- 
FPI is an aggregation of the outputs of several IT2-FPIs each associated with a specific zLevel $\left(z_{q}\right)$.

In this paper, we will construct the zT2-FPI by having $Q=2$ (i.e. having two zSlices). Hence the zT2-FPI will aggregate the output of possibly the T1-FPI (obtained possibly at zSlice level $z_{2}, y_{l_{2}}=y_{r_{2}}$ when $\gamma=1$ (as explained below) and the IT2-FPIs which use the rules structures given in Equation (2) and (4), respectively. Moreover, in the zT2-FPI controller, we will employ the same rule consequent structure, given in Equation (3). Thus, the output $\left(\dot{u}^{z T 2-F P I}\right)$ of the proposed zT2-FPI can be defined as:

$$
\dot{u}^{z T 2-F P I}=\frac{z_{1} \dot{u}^{z_{1}}+z_{2} \dot{u}^{z_{2}}}{z_{1}+z_{2}}
$$

where $\dot{u}^{z_{q}}$ is the output of an IT2-FPI controller (associated with the zSlice $z_{q}$ ) and is defined as:

$$
\dot{u}^{z_{q}}=\frac{\left(\dot{u}_{l}^{z_{q}}+\dot{u}_{r}^{z_{q}}\right)}{2}
$$

Here, $\dot{u}_{l}^{z_{q}}$ and $\dot{u}_{r}^{z_{q}}$ are the left and right end points of the type reduced set for each corresponding zSlices level and can be calculated via the KM method while the values of $z_{1}$ and $z_{2}$ are 0.5 and 1.0 , respectively.

The employed zT2-FPI controller will encompass the presented IT2-FPI structure presented in Section 2. The illustration of the employed zT2-FSs is shown in Fig.3a. In the antecedent zT2-FSs $\left(\tilde{E}_{i}^{z_{1}}, \tilde{E}_{j}^{z_{1}}\right),\left(\tilde{E}_{i}^{z_{2}}, \tilde{E}_{j}^{z_{2}}\right)$, we employed $c_{\dot{e} j}^{z_{1}}=c_{\dot{e} j}^{z_{2}}$ and $c_{e j}^{z_{1}}=c_{e j}^{z_{2}}$, i.e., the cores of IT2-FSs of each zSlices level $\left(z_{1}, z_{2}\right)$ have the same values. In order to have a convex SMF, we employ the following constraints on the heights of the lower MFs of the IT2-FPI associated with the zSlices $z_{2}$ :

$$
\begin{aligned}
& \alpha_{\dot{e} j}^{z_{2}}=\left(1-\alpha_{\dot{e} j}^{z_{1}}\right) \gamma+\alpha_{\dot{e} j}^{z_{1}} \\
& \alpha_{e i}^{z_{2}}=\left(1-\alpha_{e i}^{z_{1}}\right) \gamma+\alpha_{e i}^{z_{1}}
\end{aligned}
$$

where $\alpha_{\dot{e} j}^{z_{1}}$ and $\alpha_{e i}^{z_{1}}$ are heights of the lower MFs for the zSlices $z_{1}$ while $\alpha_{\dot{e} j}^{z_{2}}$ and $\alpha_{e i}^{z_{2}}$ are heights of the lower MFs for the zSlices $z_{2}$. Here, $\gamma$ is a tuning parameter and can take values between 0 and 1 . In order to examine how the defined $\gamma$ parameter varies the SMFs, we will examine three cases for the zT2-FSs

(i) If $\gamma=0$ then the $z_{2}$ zSlice becomes an interval set with the same uncertainty as $z_{1}$, i.e. $l_{1}=l_{2}<r_{1}=r_{2}$.Thus, an interval secondary FS will be obtained in the third dimension as illustrated in Fig. 4a.

(ii) If $0<\gamma<1$, then for the $z_{2}$ zSlice $l_{2}<r_{2}$ however the interval associated with zSlice $z_{2}$ has always a smaller interval in comparison to the interval associated with zSlice $z_{1}$ since $l_{2}>l_{1}$. Thus, a secondary FS as shown in Fig. $4 \mathrm{~b}$ will be obtained in the third dimension. The $l_{1}, l_{2}, r_{1}$ and $r_{2}$ values of this case have been shown for a zT2-FS in Fig. $3 b$.

(iii) If $\gamma=1$, then for the $z_{2}$ zSlice, $l_{2}=r_{2}$ and a secondary FS as illustrated in Fig. 4c will be obtained in the third dimension of the zT2-FS.

Thus, as it has been shown in Fig.4 for different $\gamma$ values, the
zT2-FPI controller can be constructed in three different combinations.

- If $\gamma=0$, then the zT2-FPI will be reduced to an IT2-FPI controller.

- If $0<\gamma<1$, then the zT2-FPI can be seen as collection of two IT2-FPIs associated with different FOUs $\left(r_{1}-\right.$ $l_{1}>r_{2}-l_{2}$ ).

- If $\gamma=1$, then the zT2-FPI can be seen as a collection of IT2-FPI and T1-FPI controller $\left(r_{1}-l_{1}>r_{2}-l_{2}=0\right)$.

Thus, we will first derive an IT2-FPI associated with the zSlices $\mathrm{z}_{\mathrm{q}}$ and we then will derive the output of the proposed zT2-FPI around the steady state via Equation (9) from a mathematical point of view.

\section{The Analytical Derivations for IT2-FPI Controller associated with zLevel}

In this section, we will follow the same analysis strategy presented in [1], [14] to analyze the behavior of an IT2-FPI associated with zLevel $\left(z_{q}\right)$ around the steady state from a mathematical point of view. Thus, we will investigate the region around the steady state $(e=0, \dot{e}=0, \dot{u}=0)$ bounded by the following inequalities:

$$
\begin{aligned}
& -\delta_{\dot{e}} \leq \dot{e} \leq+\delta_{\dot{e}} \\
& -\delta_{e} \leq e \leq+\delta_{e} \\
& -\delta_{\dot{u}} \leq \dot{u} \leq+\delta_{\dot{u}}
\end{aligned}
$$

where $\delta_{\dot{e}}, \delta_{e}$ and $\delta_{\dot{u}}$ are sufficiently small perturbations around the origin.

As it has been asserted in the previous subsection, we will employ the same IT2-FPI controller given in Section 2 and associated it with zLevel $\left(z_{q}\right)$. The employed IT2-FPI uses fully overlapping triangular IT2-FSs in the sense of upper and lower MFs as shown in Fig. 2. Thus for any input vector $(e, \dot{e})$ always four IT2-FSs are always fired, and the corresponding membership values around the steady state are:

$$
\begin{aligned}
& \mu_{\tilde{E}_{-1}^{z q}}=\left[\underline{\mu}_{\tilde{E}_{-1}}, \bar{\mu}_{\tilde{E}_{-1}}\right]=\left[\left(\frac{c_{\dot{e} 1}-\dot{e}}{2 c_{\dot{e} 1}}\right) \alpha_{\dot{e} 1}^{z q}, \quad\left(\frac{c_{\dot{e} 1}-\dot{e}}{2 c_{\dot{e} 1}}\right)\right] \\
& \mu_{\tilde{E}_{+1}^{z q}}=\left[\underline{\mu}_{\tilde{E}_{1}}, \bar{\mu}_{\tilde{E}_{1}}\right]=\left[\left(\frac{c_{\dot{e} 1}+\dot{e}}{2 c_{\dot{e} 1}}\right) \alpha_{\dot{e} 1}^{z_{q}}, \quad\left(\frac{c_{\dot{e} 1}+\dot{e}}{2 c_{\dot{e} 1}}\right)\right] \\
& \mu_{\tilde{E}_{-1}^{z q}}=\left[\underline{\mu}_{\tilde{E}_{-1}}, \bar{\mu}_{\tilde{E}_{-1}}\right]=\left[\left(\frac{c_{\dot{e} 1}-e}{2 c_{e 1}}\right) \alpha_{e 1}^{z_{q}}, \quad\left(\frac{c_{e 1}-e}{2 c_{e 1}}\right)\right] \\
& \mu_{\tilde{E}_{+1}^{z q}}=\left[\underline{\mu}_{\tilde{E}_{1}}, \bar{\mu}_{\tilde{E}_{1}}\right]=\left[\left(\frac{c_{\dot{e} 1}+e}{2 c_{e 1}}\right) \alpha_{e 1}^{z_{q}}, \quad\left(\frac{c_{e 1}+e}{2 c_{e 1}}\right)\right]
\end{aligned}
$$

The corresponding four rules outputs are then $R^{-1,-1}, R^{-1,1}$, $R^{1,-1}$ and $R^{1,1}$ which are defined as:

$$
\begin{gathered}
\dot{u}_{-1,-1}=-K_{P} c_{\dot{e} 1}-K_{I} c_{e 1} \\
\dot{u}_{-1,1}=-K_{P} c_{\dot{e} 1}+K_{I} c_{e 1} \\
\dot{u}_{1,-1}=K_{P} c_{\dot{e} 1}-K_{I} c_{e 1} \\
\dot{u}_{1,1}=K_{P} c_{\dot{e} 1}+K_{I} c_{e 1}
\end{gathered}
$$

The total firing interval for each rule is defined as

$$
=\left[f_{i j}^{z_{q}}, \bar{f}_{i j}^{z_{q}}\right]
$$

where $\underline{f}_{i j}^{z_{q}}$ and $\bar{f}_{i j}^{z_{q}}$ are given in Equations (23),(24), (25) and (26) (using the product operation to represent the t-norm). 


$$
\begin{aligned}
& \bar{f}_{-1,-1}^{z q}=\left[\bar{\mu}_{\tilde{E}_{-1}^{z q}} * \bar{\mu}_{\tilde{E}_{-1}^{z q}}\right]=\left[\left(\frac{c_{\dot{e} 1}-\dot{e}}{2 c_{\dot{e} 1}}\right)\left(\frac{c_{e 1}-e}{2 c_{e 1}}\right)\right], \quad \underline{f}_{-1,-1}^{z_{q}}=\left[{\underline{\tilde{E}_{-1}}}_{z^{q}} * \underline{\mu}_{\tilde{E}_{-1}^{z q}}\right]=\left[\left(\frac{c_{\dot{e} 1}-\dot{e}}{2 c_{\dot{e} 1}}\right) \alpha_{\dot{e} 1}^{z_{q}}\left(\frac{c_{\dot{e} 1}-e}{2 c_{e 1}}\right) \alpha_{e 1}^{z_{q}}\right] \\
& \bar{f}_{-1,+1}^{z_{q}}=\left[\bar{\mu}_{\tilde{E}_{-1}^{z}} * \bar{\mu}_{\tilde{E}_{+1}^{z q}}\right]=\left[\left(\frac{c_{\dot{e} 1}-\dot{e}}{2 c_{\dot{e} 1}}\right)\left(\frac{c_{e 1}+e}{2 c_{e 1}}\right)\right], \quad \underline{f}_{-1,+1}^{z_{q}}=\left[\underline{\mu}_{\tilde{E}_{-1}^{z}} * \underline{\mu}_{\tilde{E}_{+1}^{z}}^{z_{q}}\right]=\left[\left(\frac{c_{\dot{e} 1}-\dot{e}}{2 c_{\dot{e} 1}}\right) \alpha_{\dot{e} 1}^{z_{q}}\left(\frac{c_{\dot{e} 1}+e}{2 c_{e 1}}\right) \alpha_{e 1}^{z_{q}}\right] \\
& \bar{f}_{+1,-1}^{z_{q}}=\left[\bar{\mu}_{\tilde{E}_{+1}^{z q}} * \bar{\mu}_{\tilde{E}_{-1}^{z q}}\right]=\left[\left(\frac{c_{\dot{e} 1}+\dot{e}}{2 c_{\dot{e} 1}}\right)\left(\frac{c_{e 1}-e}{2 c_{e 1}}\right)\right], \quad \underline{f}_{+1,-1}^{z_{q}}=\left[\underline{\mu}_{\tilde{E}_{+1}^{z q}} * \underline{\mu}_{\tilde{E}_{-1}^{z q}}\right]=\left[\left(\frac{c_{\dot{e} 1}+\dot{e}}{2 c_{\dot{e} 1}}\right) \alpha_{\dot{e} 1}^{z_{q}}\left(\frac{c_{\dot{e} 1}-e}{2 c_{e 1}}\right) \alpha_{e 1}^{z_{q}}\right] \\
& \bar{f}_{+1,+1}^{z_{q}}=\left[\bar{\mu}_{\tilde{E}_{+1}^{z q}} * \bar{\mu}_{\tilde{E}_{+1}^{z q}}\right]=\left[\left(\frac{c_{\dot{e} 1}+\dot{e}}{2 c_{\dot{e} 1}}\right)\left(\frac{c_{e 1}+e}{2 c_{e 1}}\right)\right], \quad \underline{f}_{+1,+1}^{z_{q}}=\left[{\underline{\tilde{E}_{+1}^{z}}}^{z_{q}} * \underline{\mu}_{\tilde{E}_{+1}^{z q}}\right]=\left[\left(\frac{c_{\dot{e} 1}+\dot{e}}{2 c_{\dot{e} 1}}\right) \alpha_{\dot{e} 1}^{z_{q}}\left(\frac{c_{\dot{e} 1}+e}{2 c_{e 1}}\right) \alpha_{e 1}^{z_{q}}\right]
\end{aligned}
$$

The IT2-FPI output can be calculated as:

$$
\dot{u}^{z_{q}}=\frac{\dot{u}_{l}^{z_{q}}+\dot{u}_{r}^{z_{q}}}{2}
$$

where $\dot{u}_{l}$ and $\dot{u}_{r}$ are the left and right end points of the type reduced set, and are formulated as follows:

$$
\begin{aligned}
& \dot{u}_{l}^{{ }^{z} q}=\frac{\sum_{n=1}^{L} \bar{f}_{n}^{z_{q}} \dot{u}_{n}+\sum_{L+1}^{P} \underline{f}_{n}^{z_{q}} \dot{u}_{n}}{\sum_{n=1}^{L} \bar{f}_{n}^{z_{q}}+\sum_{L+1}^{P} \underline{f}_{n}^{z_{q}}} \\
& \dot{u}_{r}^{{ }_{q} q}=\frac{\sum_{n=1}^{R} \underline{f}_{n}^{z_{q}} \dot{u}_{n}+\sum_{R+1}^{P} \bar{f}_{n}^{z_{q}} \dot{u}_{n}}{\sum_{n=1}^{R} \underline{f}_{n}^{z_{q}}+\sum_{R+1}^{P} \bar{f}_{n}^{z_{q}}}
\end{aligned}
$$

Here, $P$ is the total number of fuzzy rules $(P=4)$ and $(L, R)$ are the switching points needed by the KM procedure [26]. When the KM method is used, the consequents $\left(\dot{u}_{i j}\right)$ need to be sorted in ascending order and their corresponding firing intervals $\left(\underline{f}_{i, j}^{z_{q}}, \bar{f}_{i, j}^{z_{q}}\right)$ must be matched. Since the sorting of the consequents $\left(\dot{u}_{i j}\right)$ depends on the values $K_{P} c_{\dot{e} 1}$ and $K_{I} c_{e 1}$, there are only three possibilities which will be examined.

1) Case-1 $\left(K_{P} c_{\dot{e} 1}>K_{I} c_{e 1}\right)$

If $K_{P} C_{\dot{e} 1}>K_{I} c_{e 1}$, then the consequents $\left(\dot{u}_{i j}\right)$ are sorted as:

$$
\dot{u}_{-1,-1}<\dot{u}_{-1,1}<0<\dot{u}_{+1,-1}<\dot{u}_{+1,+1}
$$

Since we are investigating the behavior around the steady state ( $e \approx 0, \dot{e} \approx 0, \dot{u} \approx 0)$ it can be further imposed as:

$$
\dot{u}_{-1,+1} \leq \dot{u}_{l}^{z_{q}}<\dot{u}_{r}^{z_{q}} \leq \dot{u}_{+1,-1}
$$

which indicates that the switch points of the KM must be $L=2$ and $R=2[1]$. Thus, $\dot{u}_{l}^{z_{q}}$ should be:

$\dot{u}_{l}^{z_{q}}=$

$$
\frac{\bar{f}_{-1,-1}^{z_{q}} \dot{u}_{-1,-1}+\bar{f}_{-1,+1}^{z_{q}} \dot{u}_{-1,+1}+\underline{f}_{+1,-1}^{z_{q}} \dot{u}_{+1,-1}+\underline{f}_{+1,+1}^{z_{q}} \dot{u}_{+1,+1}}{\bar{f}_{-1,-1}^{z_{q}}+\bar{f}_{-1,+1}^{z_{q}}+\underline{f}_{+1,-1}^{z_{q}}+\underline{f}_{+1,+1}^{z_{q}}}
$$

Thus, by substituting the corresponding consequent parts and the firing levels given in Equations (18-21) and (23-26), respectively into the Equation (32), we have:

$$
\begin{gathered}
\dot{u}_{l}^{z_{q}}=\frac{K_{P}\left(-c_{\dot{e} 1}^{2}+c_{e 1} \dot{e}+c_{\dot{e} 1}^{2} \alpha_{\dot{e} 1}^{z_{q}} \alpha_{e 1}^{z_{q}}+c_{e 1} \dot{e} \alpha_{\dot{e} 1}^{z_{q}} \alpha_{e 1}^{z_{q}}\right)}{\left(c_{\dot{e} 1}-\dot{e}+c_{\dot{e} 1} \alpha_{\dot{e} 1}^{z_{q}} \alpha_{e 1}^{z_{q}}+\dot{e} \alpha_{\dot{e} 1}^{z_{q}} \alpha_{e 1}^{z_{q}}\right)} \\
+\frac{K_{I}\left(c_{\dot{e} 1} e-e \dot{e}+c_{\dot{e} 1} e \alpha_{\dot{e} 1}^{z_{q}} \alpha_{e 1}^{z_{q}}+\dot{e} e \alpha_{\dot{e} 1}^{z_{q}} \alpha_{e 1}^{z_{q}}\right)}{\left(c_{\dot{e} 1}-\dot{e}+c_{\dot{e} 1} \alpha_{\dot{e} 1}^{z_{q}} \alpha_{e 1}^{z_{q}}+\dot{e} \alpha_{\dot{e} 1}^{z_{q}} \alpha_{e 1}^{z_{q}}\right)}
\end{gathered}
$$

and similarly $\dot{u}_{r}^{z_{q}}$ should be:

$$
\begin{aligned}
& \dot{u}_{r}^{z_{q}}= \\
& \frac{f_{-1,-1}^{z_{q}} \dot{u}_{-1,-1}+\underline{f}_{-1,+1}^{z_{q}} \dot{u}_{-1,+1}+\bar{f}_{+1,-1}^{z_{q}} \dot{u}_{+1,-1}+\bar{f}_{+1,+1}^{z_{q}} \dot{u}_{+1,+1}}{f_{-1,-1}^{z_{q}}+\underline{f}_{-1,+1}^{z_{q}}+\bar{f}_{+1,-1}^{z_{q}}+\bar{f}_{+1,+1}^{z_{q}}}
\end{aligned}
$$

Similarly, using the Equations (18-21) and (23-26), Equation (34) can be reformulated as

$$
\begin{gathered}
\dot{u}_{r}^{z_{q}}=\frac{K_{P}\left(c_{\dot{e} 1}^{2}+c_{e 1} \dot{e}-c_{\dot{e} 1}^{2} \alpha_{\dot{e} 1}^{z_{q}} \alpha_{e 1}^{z_{q}}+c_{e 1} \dot{e} \alpha_{\dot{e} 1}^{z_{q}} \alpha_{e 1}^{z_{q}}\right)}{\left(c_{\dot{e} 1}+\dot{e}+c_{\dot{e} 1} \alpha_{\dot{e} 1}^{z_{q}} \alpha_{e 1}^{z_{q}}-\dot{e} \alpha_{\dot{e} 1}^{z_{q}} \alpha_{e 1}^{z_{q}}\right)} \\
+\frac{K_{I}\left(c_{\dot{e} 1} e+e \dot{e}+c_{\dot{e} 1} e \alpha_{\dot{e} 1}^{z_{q}} \alpha_{e 1}^{z_{q}}-\dot{e} e \alpha_{\dot{e} 1}^{z_{q}} \alpha_{e 1}^{z_{q}}\right)}{\left(c_{\dot{e} 1}+\dot{e}+c_{\dot{e} 1} \alpha_{\dot{e} 1}^{z_{q}} \alpha_{e 1}^{z_{q}}-\dot{e} \alpha_{\dot{e} 1}^{z_{q}} \alpha_{e 1}^{z_{q}}\right)}
\end{gathered}
$$

Then, the IT2-FPI output at $z_{q}$ can be obtained by substituting Equations (33) and (35) in Equation (27)

$$
\dot{u}^{z_{q}}=\frac{\begin{array}{c}
K_{I} e\left(-\dot{e}^{2}\left(-1+\alpha_{\dot{e} 1}^{z_{q}} \alpha_{e 1}^{z_{q}}\right)^{2}+\left(c_{\dot{e} 1}+c_{\dot{e} 1} \alpha_{\dot{e} 1}^{z_{q}} \alpha_{e 1}^{z_{q}}\right)^{2}\right) \\
+K_{P} \dot{e}\left(4 c_{\dot{e} 1}^{2} \alpha_{\dot{e} 1}^{z_{q}} \alpha_{e 1}^{z_{q}}\right)
\end{array}}{-\dot{e}^{2}\left(-1+\alpha_{\dot{e} 1}^{z_{q}} \alpha_{e 1}^{z_{q}}\right)^{2}+\left(c_{\dot{e} 1}+c_{\dot{e} 1} \alpha_{\dot{e} 1}^{z_{q}} \alpha_{e 1}^{z_{q}}\right)^{2}}
$$

Then, Equation (36) can be reformulated as:

$$
\dot{u}^{z_{q}}=K_{P_{-} e q}^{z_{q}} \dot{e}+K_{I_{-} e q}^{z_{q}} e
$$

where

$$
\begin{gathered}
K_{P_{-} e q}^{z_{q}}=K_{P} \delta_{1}^{z_{q}}, \quad K_{I_{-} e q}^{z_{q}}=K_{I} \beta_{1}^{z_{q}} \\
\delta_{1}^{z_{q}}=\left(\begin{array}{c}
\frac{4 c_{\dot{e} 1}^{2} \alpha_{\dot{e} 1}^{z_{q}} \alpha_{e 1}^{z_{q}}}{-\dot{e}^{2}\left(-1+\alpha_{\dot{e} 1}^{z_{q}} \alpha_{e 1}^{z_{q}}\right)^{2}+\left(c_{\dot{e} 1}+c_{\dot{e} 1} \alpha_{\dot{e} 1}^{z_{q}} \alpha_{e 1}^{z_{q}}\right)^{2}} \\
\beta_{1}^{z_{q}}=1
\end{array}\right)
\end{gathered}
$$

Here, $K_{P_{-} e q}^{z_{q}}$ and $K_{I_{-} e q}^{z_{q}}$ are the equivalent proportional and integral gains of the resulting IT2-FPI controller associated with zLevel $\left(z_{q}\right)$, respectively.

2) Case-2 $\left(K_{P} c_{\dot{e} 1}<K_{I} c_{e 1}\right)$

If $K_{P} c_{\dot{e} 1}<K_{I} c_{e 1}$, then the consequents $\left(\dot{u}_{i j}\right)$ are sorted as:

$$
\dot{u}_{-1,-1}<\dot{u}_{1,-1}<0<\dot{u}_{-1,1}<\dot{u}_{1,1}
$$

Since we are investigating the behavior around the steady state, it can be further imposed as:

$$
\dot{u}_{1,-1} \leq \dot{u}_{l}^{z_{q}}<\dot{u}_{r}^{z_{q}} \leq \dot{u}_{-1,1}
$$

which indicates that the switch points of the KM are $L=2$ and $R=2$ [1]. Similarly in the Case- $1, \dot{u}_{l}^{z_{q}}$ and $\dot{u}_{r}^{z_{q}}$ are defined as: $\dot{u}_{l}^{z_{q}}=$

$$
\begin{gathered}
\frac{\bar{f}_{-1,-1}^{z_{q}} \dot{u}_{-1,-1}+\bar{f}_{+1,-1}^{z_{q}} \dot{u}_{1,-1}+f_{-1,+1}^{z_{q}} \dot{u}_{-1,1}+f_{+1,+1}^{z_{q}} \dot{u}_{1,1}}{\bar{f}_{-1,-1}^{z_{q}}+\bar{f}_{+1,-1}^{z_{q}}+\bar{f}_{-1,+1}^{z_{q}}+f_{+1,+1}^{z_{q}}} \\
\dot{u}_{l}^{z_{q}}=\frac{K_{P}\left(c_{e 1} \dot{e}-\dot{e} e+c_{e 1} \dot{e} \alpha_{\dot{e} 1}^{z_{q}} \alpha_{e 1}^{z_{q}}+\dot{e} e \alpha_{\dot{e} 1}^{z_{q}} \alpha_{e 1}^{z_{q}}\right)}{\left(c_{e 1}-e+c_{e 1} \alpha_{\dot{e} 1}^{z_{q}} \alpha_{e 1}^{z_{q}}+e \alpha_{\dot{e} 1}^{z_{q}} \alpha_{e 1}^{z_{q}}\right)}+ \\
+\frac{K_{I}\left(-c_{e 1}^{2}+c_{\dot{e} 1} e+c_{e 1}^{2} \alpha_{\dot{e} 1}^{z_{q}} \alpha_{e 1}^{z_{q}}+c_{\dot{e} 1} e \alpha_{\dot{e} 1}^{z_{q}} \alpha_{e 1}^{z_{q}}\right)}{\left(c_{e 1}-e+c_{e 1} \alpha_{\dot{e} 1}^{z_{q}} \alpha_{e 1}^{z_{q}}+e \alpha_{\dot{e} 1}^{z_{q}} \alpha_{e 1}^{z_{q}}\right)}
\end{gathered}
$$

and 


$$
\begin{aligned}
& \dot{u}_{r}^{z_{q}}= \\
& \frac{\underline{f}_{-1,-1}^{z_{q}} \dot{u}_{-1,-1}+\underline{f}_{+1,-1}^{z_{q}} \dot{u}_{+1,-1}+\bar{f}_{-1,+1}^{z_{q}} \dot{u}_{-1,+1}+\bar{f}_{+1,+1}^{z_{q}} \dot{u}_{+1,+1}}{f_{-1,-1}^{z_{q}}+\underline{f}_{+1,-1}^{z_{q}}+\bar{f}_{-1,+1}^{z_{q}}+\bar{f}_{+1,+1}^{z_{q}}} \\
& \dot{u}_{r}^{z_{q}}=\frac{K_{P}\left(c_{e 1} \dot{e}+\dot{e} e+c_{e 1} \dot{e} \alpha_{\dot{1} 1}^{z_{q}} \alpha_{e 1}^{z_{q}}-\dot{e} e \alpha_{\dot{e} 1}^{z_{q}} \alpha_{e 1}^{z_{q}}\right)}{\left(c_{e 1}+e+c_{e 1} \alpha_{\dot{e} 1}^{z_{q}} \alpha_{e 1}^{z_{q}}-e \alpha_{\dot{e} 1}^{z_{q}} \alpha_{e 1}^{z_{q}}\right)}+ \\
& \quad+\frac{K_{I}\left(c_{e 1}^{2}+c_{\dot{e} 1} e-c_{e 1}^{2} \alpha_{\dot{e} 1}^{z_{q}} \alpha_{e 1}^{z_{q}}+c_{\dot{e} 1} e \alpha_{\dot{e} 1}^{z_{q}} \alpha_{e 1}^{z_{q}}\right)}{\left(c_{e 1}+e+c_{e 1} \alpha_{\dot{e} 1}^{z_{q}} \alpha_{e 1}^{z_{q}}-e \alpha_{\dot{e} 1}^{z_{q}} \alpha_{e 1}^{z_{q}}\right)}
\end{aligned}
$$

Then, the output of the IT2-FPI can be obtained by substituting Equations (43) and (45) in Equation (27)

$$
\dot{u}=\frac{K_{P} \dot{e}\left(-e^{2}\left(-1+\alpha_{\dot{e} 1}^{z_{q}} \alpha_{e 1}^{z_{q}}\right)^{2}+\left(c_{e 1}+c_{e 1} \alpha_{\dot{e} 1}^{z_{q}} \alpha_{e 1}^{z_{q}}\right)^{2}\right)}{+K_{I} e\left(4 c_{e 1}^{2} e \alpha_{\dot{e} 1}^{z_{q}} \alpha_{e 1}^{z_{q}}\right)}
$$

Then, Equation (46) can be reformulated as:

$$
\dot{u}^{z_{q}}=K_{P_{-} e q}^{z_{q}} \dot{e}+K_{I_{-} e q}^{z_{q}} e
$$

where

$$
\begin{aligned}
& K_{P_{-} e q}^{z_{q}}=K_{P} \delta_{2}^{z_{q}}, \quad K_{I_{-} e q}^{z_{q}}=K_{I} \beta_{2}^{z_{q}} \\
& \delta_{2}^{z_{q}}=1 \\
& \beta_{2}^{z_{q}}=\left(\frac{4 c_{e 1}^{2} \alpha_{\dot{e} 1}^{z_{q}} \alpha_{e 1}^{z_{q}}}{-e^{2}\left(-1+\alpha_{\dot{e} 1}^{z_{q}} \alpha_{e 1}^{z_{q}}\right)^{2}+\left(c_{e 1}+c_{e 1} \alpha_{\dot{e} 1}^{z_{q}} \alpha_{e 1}^{z_{q}}\right)^{2}}\right)
\end{aligned}
$$

Here, $K_{P_{-} e q}$ and $K_{I_{-} e q}$ are the equivalent proportional and integral gains of the resulting IT2-FPI controller associated with zLevel $\left(z_{q}\right)$, respectively.

$$
\begin{aligned}
& \text { 3) Case-3 }\left(K_{P} c_{\dot{e} 1}=K_{I} c_{e 1}\right) \\
& \text { If } K_{P} c_{\dot{e} 1}=K_{I} c_{e 1} \text {, then } \dot{u}_{-1,+1}=\dot{u}_{+1,-1}=0 \text { and } \\
& \dot{u}_{-1,-1}<\dot{u}_{+1,-1}=0=\dot{u}_{-1,+1}<\dot{u}_{+1,+1}
\end{aligned}
$$

Thus, there are more than one possible switching point for the KM which are $R, L \in\{1,2,3\}$. Therefore, similar analysis cannot be performed here since the switching points cannot be predetermined [1]. Thus, we will not examine the case $K_{P} \dot{e}_{1}=K_{I} e_{1}$ in this study. However, this does not affect the above analysis.

\section{The Analytical Derivations for zT2-FPI Controller}

In this subsection, we will use the derived equations of the IT2-FPI associated with zLevel $\left(z_{q}\right)$ to express the output of the zT2-FPI around the steady state from a mathematical point of view. As it has been presented in the subsection 3.2, the output of the zT2-FPI is an aggregation of the outputs of IT2FPIs (and maybe T1-FPI) each associated with a specific zLevel $\left(z_{q}\right)$. Then, the output of the zT2-FPI can be obtained by substituting Equation (37) (for Case-1) or Equation (47) (for Case-2) in Equation (9):

$$
\dot{u}^{Z T 2-F P I}=\frac{z_{1}\left(K_{P_{-} e q}^{Z_{1}} \dot{e}+K_{I_{-} e q}^{Z_{1}} e\right)+z_{2}\left(K_{P_{-} e q}^{Z_{2}} \dot{e}+K_{I_{-} e q}^{Z_{2}} e\right)}{z_{1}+z_{2}}
$$

Then, Equation (51) can be reformulated as:

$$
\dot{u}^{Z T 2-F P I}=K_{P_{-} e q} \dot{e}+K_{I_{-} e q} e
$$

where

$$
K_{P_{-} e q}=\frac{\left(z_{1} K_{P_{-} e q}^{Z_{1}}+z_{2} K_{P_{\_} e q}^{Z_{2}}\right)}{z_{1}+z_{2}} \quad K_{I_{-} e q}=\frac{\left(z_{1} K_{I_{-} e q}^{Z_{1}}+z_{2} K_{I_{-} e q}^{Z_{2}}\right)}{z_{1}+z_{2}}
$$

Here, $K_{P_{-} e q}$ and $K_{I_{-} e q}$ are the equivalent Proportional gain and the equivalent Integral gain of the resulting zT2-FPI controller, respectively. It should be noted that the $K_{P_{-} e q}$ and $K_{I_{-} e q}$ can be defined in two different ways since equalities of $K_{P_{-} e q}^{z_{q}}$ and $K_{I_{-} e q}^{z_{q}}$ depends on the values $K_{P} c_{\dot{e} 1}$ and $K_{I} c_{e 1}$. Thus, for $K_{P} c_{\dot{e} 1}>K_{I} c_{e 1}$ (Case-1) Equation (38) should be employed while for $K_{P} c_{\dot{e} 1}<K_{I} c_{e 1}$ (Case-2) Equation (48) should be employed to obtain the equivalent gains of the zT2-FPI.

For Case-1, then the equivalent gains are

$$
K_{P_{-} e q}^{Z_{1}}=K_{P} \delta_{1}^{Z_{1}}\left(e, c_{e 1}, \alpha_{\dot{e} 1}^{Z_{1}}, \alpha_{e 1}^{Z_{1}}\right) \quad K_{I_{-} e q}^{Z_{1}}=K_{I} \text { for zLevel } z_{1}
$$$$
K_{P_{-} e q}^{z_{2}}=K_{P} \delta_{1}^{z_{2}}\left(e, c_{e 1}, \alpha_{\dot{e} 1}^{Z_{2}}, \alpha_{e 1}^{z_{2}}\right) \quad K_{I_{-} e q}^{z_{2}}=K_{I} \quad \text { for zLevel } z_{2}
$$

Substituting Equation (11) and Equation (12) into Equation (55) where $\gamma=\gamma_{\dot{e}}=\gamma_{e}, K_{P_{-} e q}^{z_{2}}$ can be reformulated as function

$$
K_{P_{-} e q}^{z_{2}}=K_{P} \delta_{1}^{z_{2}}\left(e, c_{e 1}, \alpha_{\dot{e} 1}^{z_{1}}, \alpha_{e 1}^{z_{1}}, \gamma\right)
$$

Thus Equation (53) can be reformulated for Case-1 as follows:

$$
K_{P_{-} e q}=\frac{\left(z_{1} \delta_{1}^{z_{1}}\left(e, c_{e 1}, \alpha_{e 1}^{z_{1}}, \alpha_{e 1}^{z_{1}}\right)+z_{2} \delta_{1}^{z_{2}}\left(e, c_{e 1}, \alpha_{e 1}^{z_{1}}, \alpha_{e 1}^{z_{1}}, \gamma\right)\right)}{z_{1}+z_{2}} K_{P}
$$

Similarly for Case-2, then the equivalent gains are $K_{P_{-} e q}^{Z_{1}}=K_{P}, \quad K_{I_{-} e q}^{z_{1}}=K_{I} \beta_{2}^{z_{1}}\left(e, c_{e 1}, \alpha_{\dot{e} 1}^{z_{1}}, \alpha_{e 1}^{z_{1}}\right)$ for zLevel $z_{1}$ $K_{P_{-} e q}^{z_{2}}=K_{P}, \quad K_{I_{-} e q}^{z_{2}}=K_{I} \beta_{2}^{Z_{2}}\left(e, c_{e 1}, \alpha_{\dot{e} 1}^{Z_{2}}, \alpha_{e 1}^{Z_{2}}\right) \quad$ for zLevel $z_{2}$ Similarly using Equation (11) and Equation (12), $K_{I_{-} e q}^{z_{2}}$ can be reformulated as function

$$
K_{I_{-} e q}^{Z_{2}}=K_{I} \beta_{2}^{Z_{2}}\left(e, c_{e 1}, \alpha_{\dot{e} 1}^{z_{1}}, \alpha_{e 1}^{z_{1}}, \gamma\right)
$$

Thus Equation (60) can be reformulated for Case-2 as follows:

$$
K_{I_{-} e q}=\frac{K_{I}\left(z_{1} \beta_{2}^{z_{1}}\left(e, c_{e 1}, \alpha_{\dot{e} 1}^{z_{1}}, \alpha_{e 1}^{z_{1}}\right)+z_{2} \beta_{2}^{z_{2}}\left(e, c_{e 1}, \alpha_{\dot{e} 1}^{z_{1}}, \alpha_{e 1}^{z_{1}}, \gamma\right)\right)}{z_{1}+z_{2}}
$$

\section{EFFECT OF THE SMF ON THE TYPE-2 FUZZY PI CONTROLLER PERFORMANCE}

In this section, we will try to illustrate the effect of the SMF on controller performance. As it has been asserted in Subsection 3.2, the SMF of a T2-FS can be constructed in three different ways with respect to the defined parameter $\gamma$. Thus, we will first investigate the effect of the size of the SMF when $\gamma=0$ (interval SMF) and then the effect the shape of the SMF for a fixed SMF size $\left(u_{0}\right)$ when $0<\gamma \leq 1$ (to have the shapes in Fig. $4 \mathrm{~b}$ or $4 \mathrm{c}$ ) on the control performance. For both cases, we will analyze the variations of the equivalent PI gains and their effects on the control performance. In order to have an easier analysis, we will examine the control actions of the zIT2-FPI by presenting the equivalent controller gain $\left(K_{c_{-} e q}\right)$ and integral time constant $\left(T_{i_{-} e q}\right)$ of the PI controller which are defined as:

$$
K_{C_{-} e q}=K_{P_{-} e q}, \quad T_{i_{-} e q}=K_{P_{-} e q} / K_{I_{-} e q}
$$

We would like to remind that, a decreased value of $K_{c_{-} e q}$ gives less proportional action and slower control action, i.e. the damping of the system will increase; while a decreased value of $T_{i_{-} e q}$ provides more integral action and a faster control performance, i.e. the damping of the system will decrease [28]. We will examine the control performances of the zIT2FPI controllers for the following system:

$$
G(s)=\frac{K}{(\tau s+1)^{2}}
$$


where $K$ is the gain and $\tau$ is the time constant of the system. The nominal system parameters are $K=1$ and $\tau=1$. We will also examine the robustness of the zT2-FPI controller when the system is perturbed $(K=1.9, \tau=0.7)$ (i.e. to enable examining the system response against parameter variations). We will define each input domain $(e$ and $\dot{e})$ of the zT2-FPI controller with two T2-FSs as shown in Fig. 3, where $c_{\dot{e} 1}=$ $c_{\dot{e} 1}^{z_{1}}=c_{\dot{e} 1}^{z_{2}}=1, c_{e 1}=c_{e 1}^{z_{1}}=c_{e 1}^{z_{2}}=1, \quad \alpha^{z_{1}}=\alpha_{\dot{e} 1}^{z_{1}}=\alpha_{e 1}^{z_{1}}$ and $\alpha^{z_{2}}=\alpha_{\dot{e} 1}^{z_{2}}=\alpha_{e 1}^{z_{2}}$. In order to examine how the SMF of the T2-FSs affects the transient state performances, a unit step reference is applied. Moreover, input and output disturbances with the magnitudes of " 0.2 " have been applied in $20^{\text {th }}$ and $40^{\text {th }}$ seconds, respectively to observe the disturbance rejection performances.

\section{A. Effect of the size of the FOU on the controller performance}

In this subsection, we will investigate the effect of the size of the FOU on the control performance when $\gamma=0$, (when an interval FS is employed as the SMF). As it has been asserted in subsection 3.2, the zT2-FPI will reduce to an IT2-FPI structure. Thus, the heights of the lower MF of each zSlice will be equal, i.e. $\alpha=\alpha^{z_{1}}=\alpha^{z_{2}}$ when $\gamma=0$. Moreover, as it has been derived in subsection 3.3, the effect of the FOU on the equivalent controller gains varies with respect to the baseline T1-FPI controller parameters.

1) Case-1 $\left(K_{P} c_{\dot{e} 1}>K_{I} c_{e 1}\right)$

When $\gamma=0$, Equation (57) will simplify to

$$
K_{P_{-} e q}=K_{P} \delta_{1}, \quad K_{I_{-} e q}=K_{I} \beta_{1}
$$

where

$$
\delta_{1}=\left(\frac{4 c_{\dot{e} 1}^{2} \alpha^{2}}{-\dot{e}^{2}\left(-1+\alpha^{2}\right)^{2}+\left(c_{\dot{e} 1}+c_{\dot{e} 1} \alpha^{2}\right)^{2}}\right), \quad \beta_{1}=1
$$

Consequently, the equivalent controller gain and integral time constant of the zIT2-FPI reduce to:

$$
K_{C_{-} e q}=K_{P} \delta_{1}, \quad T_{i_{-} e q}=\frac{K_{P} \delta_{1}}{K_{I}}
$$

It can be seen from Equation (66) that, the zT2-FPI realizes a non-linear PI where both equivalent controller gain $\left(K_{c_{-} e q}\right)$ and integral time constant $\left(T_{i_{-} e q}\right)$ is varying as a result of the extra degrees of freedom provided by the FOUs. It can be observed that:

1) When $K_{P} c_{\dot{e} 1}>K_{I} c_{e 1}$, only $\delta_{1}$ is a function of $\dot{e}, c_{\dot{e} 1}$, and $\alpha$ while $\beta_{1}$ is a constant value around the origin $(e=0, \dot{e}=0, \dot{u}=0)$. To illustrate the variation of $\delta_{1}\left(\dot{e}, c_{\dot{e} 1}, \alpha\right)$, we will plot the change for a certain interval of $\dot{e}$ with four fixed values $\alpha=\{0.5,0.6,0.7,0.8\}$ (the same shapes are repeatable for various values of $\alpha$ ) which is illustrated in Fig.5a. We would like to underline that the given intervals of $\dot{e} \in[-0.5,0.5]$ has been chosen only for illustrative purposes so that the effect of the FOU on the gains can be seen clearly. It should be noted that the derivation in Equation (30) is valid on for the intervals which satisfy Equation (13).

2) When the FOUs increase, then $\delta_{1}\left(\dot{e}, c_{\dot{e} 1}, \alpha\right)$ decreases for the same $\dot{e}$ as shown in Fig.5a. It can be seen that since the $\delta_{1}\left(\dot{e}, c_{\dot{e} 1}, \alpha\right)$ is smaller than unity around the steady state, thus the equivalent controller gain $\left(K_{c_{-} e q}\right)$ and integral time constant $\left(T_{i_{-} e q}\right)$ will always be smaller than the baseline T1-FPI controller ones $\left(K_{c}, T_{i}\right)$. However, since both $K_{c_{-} e q}$ and $T_{i_{-} e q}$ decrease, a larger FOU might decrease the damping of the zT2-FPI controller around the steady state, and hence reduces rise time while this may also increase the overshoot. Thus, the fuzzy control system may even become unstable in the presence of unmodelled dynamics, nonlinearities and uncertainties.

In the rest of the paper, we will not focus on Case-1 since in conventional or self-tuning fuzzy controller design strategies usually the aim is to decrease the integral action sufficiently around the steady state to have robust system performance against noises and parameter variations [16], [29], hence we will mainly focus in this paper on Case-2 mentioned in the following subsection.

2) Case-1 $\left(K_{P} c_{\dot{e} 1}<K_{I} c_{e 1}\right)$

When $\gamma=0$, Equation (61) will simplify to

$$
K_{P_{-} e q}=K_{P} \delta_{2} \quad K_{I_{-} e q}=K_{I} \beta_{2}
$$

where

$$
\delta_{2}=1 \quad \beta_{2}=\left(\frac{4 c_{e 1}^{2} \alpha^{2}}{-e^{2}\left(-1+\alpha^{2}\right)^{2}+\left(c_{\dot{e} 1}+c_{e 1} \alpha^{2}\right)^{2}}\right)
$$

Consequently, the equivalent controller gain and integral time constant of the zIT2-FPI reduce to:

$$
K_{c_{-} e q}=K_{P}, \quad T_{i_{-} e q}=\frac{K_{P}}{K_{I} \beta_{2}}
$$

It can be concluded that, the zT2-FPI realizes a non-linear PI where only the integral time constant $\left(T_{i_{-} e q}\right)$ is varying as a result of the extra degrees of freedom provided by the FOUs. Thus, it can be observed that:

1) When $K_{P} \dot{e}_{1}<K_{I} e_{1}$, only $\beta_{2}$ is a function of $e, c_{e 1}$ and $\alpha$ while $\delta_{2}$ is a constant value around the origin $(e=0, \dot{e}=$ $0, \dot{u}=0)$. To illustrate the variation of $\beta_{2}\left(e, c_{e 1}, \alpha\right)$, we will plot the change for a certain interval of $e$ with four fixed values $\alpha=0.5,0.6,0.7$ and 0.8 which is illustrated in Fig.5b. It should be noted that the given interval of $e \in[-0.5,0.5]$ has been chosen only for illustrative purposes so that the effect of the FOU on the gains can be seen clearly.

2) As shown in Fig.5b, when the FOUs increase then $\beta_{2}\left(e, c_{e 1}, \alpha\right)$ decrease for the same $e$. It should be noted that since the $\beta_{2}\left(e, c_{e 1}, \alpha\right)$ is smaller than unity around the steady state, the equivalent integral time constant $\left(T_{i \_e q}\right)$ will always be larger than the baseline T1-FPI controller ones $\left(T_{i}\right)$. Consequently, larger FOUs will result in larger integral time constant gain $\left(T_{i_{-} e q}\right)$ around the origin which will increase the damping of zT2-FPI in comparison to the baseline T1-FPI controller ones. Since the equivalent integral action decreases as the system output reaches the reference signal, this will increase the damping and may decrease the overshoot and the resulting zT2 FPI controller is potentially more robust against parameter variations around the steady state. However, increasing the size of the FOU of the type- 2 fuzzy system might slow the system response in comparison with the type-1 fuzzy counterpart. Thus, the 
disturbance rejection performance might be worse (too slow) since a relatively small control signal change will be generated around the steady state as the FOU increases. Consequently, a proper tuning of $\alpha$ might give the opportunity increase the speed of the control system while preserving the robustness against parameter variations around the steady state.

To examine how the size of the FOUs affect the control system performances, the control performance of the fuzzy controllers for the cases $\alpha=1$ (i.e. a T1-FPI controller) and $\alpha=0.2$ (i.e. an IT2-FPI controller since $\gamma=0$ ) are illustrated in Fig. 6a for the nominal system while in Fig. $6 \mathrm{~b}$ for the perturbed system. For illustrative purposes, the parameters of the zT2-FPI are set as $K_{P}=0.2023$ and $K_{I}=0.46$ such that $K_{P} c_{\dot{e} 1}<K_{I} c_{e 1}$ is satisfied. As it can be clearly seen a larger FOU (i.e., a smaller $\alpha$ value) increases the damping of the zT2-FPI, and reduces the overshoots and oscillations in the transient state response. However, as the FOU increases, the system response becomes slower and the disturbance rejection times increases (as shown in time steps $20^{\text {th }}$ and $40^{\text {th }} \mathrm{sec}$ in Fig. 6a and Fig.6b) since a much smoother control signal will be generated around the steady sate since the integral equivalent gain $K_{I_{-} e q}$ is smaller in comparison to its type-1 counterpart.

\section{B. Effect of the shape of the SMF on the controller performance}

In this subsection, we will investigate the effect the shape of the SMF for a fixed FOU size when $0 \leq \gamma<1$ (resulting in the SMF shapes in Fig. $4 \mathrm{~b}$ and $4 \mathrm{c}$ ) on the controller performance. As it has been asserted, the zT2-FPI will be constructed as a collection of two IT2-FPI controllers. Moreover, the effect of the shape of the SMF on the equivalent controller gains varies with respect to the baseline T1-FPI controller parameters. However, as we have underlined at the end of subsection (4.1.1), we will only focus on Case-2 $\left(K_{P} c_{\dot{e} 1}<K_{I} c_{e 1}\right)$ where the integral action is decreased around the steady state to have robust performance against noises and parameter variations. Thus, the consequent parameters of the zT2-FPI are set as $K_{P}=0.2023$ and $K_{I}=$ 0.46. Since we employ $\alpha_{\dot{e} 1}^{z_{1}}=\alpha_{e 1}^{z_{1}}=\alpha$ and $\alpha^{z_{2}}=\alpha_{\dot{e} 1}^{z_{2}}=\alpha_{e 1}^{z_{2}}$, Equation (11) and (12) simplifies to $\alpha^{z_{2}}=(1-\alpha) \gamma+\alpha$. Consequently, Equation (61) will simplify to

$$
\begin{gathered}
K_{P_{-} e q}=K_{P} \delta_{3} \quad K_{I_{-} e q}=K_{I} \beta_{3} \\
\delta_{3}=1 \quad \beta_{3}=\frac{\left(z_{1} \beta_{2}^{z_{1}}\left(e, c_{e 1}, \alpha\right)+z_{2} \beta_{2}^{z_{2}}\left(e, c_{e 1}, \alpha, \gamma\right)\right)}{z_{1}+z_{2}}
\end{gathered}
$$

where

$$
\beta_{2}^{z_{1}}\left(e, c_{e 1}, \alpha\right)=\left(\frac{4 c_{e 1}^{2} \alpha^{2}}{-e^{2}\left(-1+\alpha^{2}\right)^{2}+\left(c_{\dot{e} 1}+c_{e 1} \alpha^{2}\right)^{2}}\right)
$$

$\beta_{2}^{Z_{2}}\left(e, c_{e 1}, \alpha, \gamma\right)=$

$\left(\frac{4 c_{e 1}^{2}(\gamma(1-\alpha)+\alpha)^{2}}{-e^{2}\left(-1+(\gamma(1-\alpha)+\alpha)^{2}\right)^{2}+\left(c_{\dot{e} 1}+c_{e 1}(\gamma(1-\alpha)+\alpha)^{2}\right)^{2}}\right)$

Consequently, the equivalent controller gain and integral time constant of the zIT2-FPI reduce to:

$$
K_{c_{-} e q}=K_{P}, \quad T_{i_{-} e q}=\frac{K_{P}}{K_{I} \beta_{3}}
$$

It can be concluded that, the zT2-FPI for $0<\gamma \leq 1$ realizes a non-linear PI where only the integral time constant $\left(T_{i_{-} e q}\right)$ is varying as a result of the shape of the SMFs varies. It can be observed that:

1) Only $\beta_{3}$ is a function of $e, c_{e 1}, \alpha$ and $\gamma$ while $\delta_{3}$ is a constant value around the origin $(e=0, \dot{e}=0, \dot{u}=0)$. The variation of $\beta_{3}\left(e, c_{e 1}, \alpha, \gamma\right)$ with respect to $\gamma$ is illustrated in Fig.5c for a certain interval of $e$ is illustrated in with four fixed values $\gamma=0,0.3,0.6$ and 1.0 where $\alpha$ is fixed and set as $\alpha=0.5$ (the size of the $\operatorname{FOU}\left(u_{0}\right)$ is fixed). Here also the case $\gamma=0$ (where the zT2-FPI reduces to an IT2-FPI) is also presented for comparison. The given interval of $e \in[-0.5,0.5]$ has been chosen only for illustrative purposes so that gain variation can be seen clearly.

2) As shown in Fig.5c, the equivalent integral time constant $\left(T_{i \_e q}\right)$ will always bigger than the IT2-FPI (zT2-FPI with $\gamma=0$ ) one's but is always smaller than the baseline T1FPI one's. Consequently, a proper tuning of the defined parameter $\gamma$ might give the opportunity increase the speed of the control system while preserving the robustness against parameter variations around the steady state.

In order to validate these observations, we will briefly examine the effect of the tuning parameter $\gamma$ on the control performance for the process given in Equation (63) and we will employ $\alpha=0.2$. In order to examine how the shape of the SMF effects the control performances with respect to the parameter $\gamma$, the step response and disturbance rejection performances are examined for the cases $\gamma=1$ (a secondary FS as shown in Fig4c), $\gamma=0.1$ (a secondary FS as shown in Fig4b) and $\gamma=0$ (Interval secondary FS, i.e. an IT2-FPI). The control performances of the zT2-FPI control systems are given in Fig. 7a for the nominal system while Fig. 7b shows the control performances for the perturbed system. As it can be seen, the zT2-FPI structure with the $\gamma=0.1$ value decreased the settling time with a small overshoot while enhancing the disturbance rejection performances and preserving the robustness against parameter variations in comparison with the IT2-FPI $(\gamma=0)$. Thus, the tuning parameter $\gamma$ of the zT2-FS gives the opportunity to the control system to have a fast rise time and a small overshoot as well as a short settling time while enhancing the disturbance rejection performance.

\section{The ZSlices Based On-Line Tuning Strategy}

In this section, we will propose two tuning mechanism for the zT2-FPI structure to improve the transient and disturbance rejection performances while preserving the robustness of an IT2-FPI controller. As we have illustrated in Section 4. The size of FOU and the shape of the SMF within a zT2-FPI controller have an important effect on transient system and disturbance rejection performance. In the control engineering literature, it has been stated that objectives such as transient state performance, robustness against parameter variations, input and output disturbances and noise should be taken in account while designing the controllers [17]. However, a controller might not be able to improve both the robustness and the control performance at the same time. This problem is 
usually solved by a trade-off between control performance and robustness [18]. Thus, as we have illustrated in the Section 4, determining carefully the size or shape of the third dimension of T2-FSs might be a good opportunity to enhance both the transient state and disturbance rejection performance of the zT2-FPI controller while preserving the robustness against parameter variations, noise and uncertainties. Considering the mathematical derivations presented in Section 4, an appropriate $\gamma$ (which determines the shape of the SMF) or the FOU size (where $\gamma=0$ ) of the zT2-FPI can be generated in an online manner to have a robust fast system response while enhancing the disturbance rejection performances. Thus, we will propose first an online tuning mechanism to generate the FOU size $(\gamma=0)$ of the zT2-FPI controller. We will then propose an online tuning mechanism to generate an appropriate $\gamma(0<\gamma \leq 1)$ of the zT2-FPI controller.

\section{A. Online tuning strategy for the FOU size of the zT2-FPI controller (FT-zT2-FPI)}

In this subsection, we will present an online tuning mechanism to generate the FOU size $(\gamma=0)$ based on the derivations in Subsection (4.1). Since $\gamma=0$, Equation (11) and (12) will reduce to $\alpha_{\dot{e}}^{z_{1}}=\alpha_{\dot{e}}^{z_{2}}$ and $\alpha_{e}^{z_{1}}=\alpha_{e}^{z_{2}}$. We will first define the following equations for the tuning strategy of the FOU size:

$$
\begin{aligned}
& \alpha_{\dot{e}_{\text {new }}}^{z_{1}}=\left(1-\alpha_{\dot{e}_{0}}^{z_{1}}\right) \rho+\alpha_{\dot{e}_{0}}^{z_{1}} \\
& \alpha_{e_{\text {new }}}^{z_{1}}=\left(1-\alpha_{e_{0}}^{z_{1}}\right) \rho+\alpha_{e_{0}}^{z_{1}}
\end{aligned}
$$

where $\rho$ is a new tuning parameter $(0 \leq \rho \leq 1)$. Consequently, the tuning interval for $\alpha_{\dot{e}_{n e w}}^{z_{1}}$ is then $\alpha_{\dot{e}_{0}}^{z_{1}} \leq$ $\alpha_{\dot{e}_{\text {new }}}^{z_{1}} \leq 1$ while for $\alpha_{e_{\text {new }}}^{z_{1}}$ the tuning interval is $\alpha_{e_{0}}^{z_{1}} \leq$ $\alpha_{e_{\text {new }}}^{z_{1}} \leq 1$. Here, $\alpha_{\dot{e}_{0}}^{z_{1}}$ and $\alpha_{e_{0}}^{z_{1}}$ are the lower bounds of the heights of the lower MFs (which will set equal to the ones of the OIT2-FPI in Section 6). Thus, the zT2-FPI controller will become a T1-FPI for $\rho=1$, while for $0 \leq \rho<1$ will converge to IT2-FPI. Thus, an appropriate $\rho$ (which determines the FOU size) can be generated to speed the system response while preserving the robustness as it has been asserted in subsection (4.1). In this context, we will use the feedback error $(e)$ for tuning the parameter in an online manner. Since the interval for $\rho$ is within the range $[0,1]$, the interval of the error $[-1,1]$ is mapped to the interval $[0,1]$ as follows:

$$
\begin{gathered}
\sigma_{1}(e)=A b s(e) \\
\sigma_{2}(e)=1-A b s(e)
\end{gathered}
$$

One of these functions can be directly assigned as the tuning parameter $\rho$. In this context, the step response of the closed loop system is divided into four main regions as illustrated in Fig. 8 where $r$ is the reference signal and $y$ is the system output. The defined tuning parameter should take different values at each region to achieve a satisfactory performance. The following heuristic tuning strategy can be used:

- At region 1, the feedback error value is positive and the system response approaches the set-point, therefore the damping of control system should be increased in time to accelerate the system response sufficiently and prevent possible overshoot. Thus, the value of $\rho$ should be decreased in time to accelerate the system response sufficiently. Thus, the tuning parameter can be tuned as

$$
\rho=\varphi * \sigma_{2}(e)
$$

Here, the value of " $\varphi$ " is taken to be " 1 " for all the regions except the first region. Since the error value possesses its extreme value at the region 1 , the value of " $\varphi$ " can take values like 0.4 or 0.5 so as to prevent the possible overshoots in the system response.

- At region 2, the system error value is negative and the system response drifts away from the set-point therefore the damping of control system should be decreased to reduce the overshoot. Thus, $\rho$ should be increased sufficiently as follows:

$$
\rho=\sigma_{1}(e)
$$

- At region 3, the system error value is negative and the system response approaches the set-point, therefore the damping of control system should be decreased in time to accelerate the system response sufficiently. Thus, the tuning parameter is tuned as

$$
\rho=\sigma_{1}(e)
$$

- At region 4 , the system error value is positive and the system response drifts away from the set-point, therefore the damping of control system should be decreased to reduce the undershoot. Thus, $\rho$ should be increased sufficiently as;

$$
\rho=\sigma_{2}(\mathrm{e})
$$

If the error $(e)$ and change of error $(\dot{e})$ has entered and remained within a specified error band, such as $2 \%$, then the tuning mechanism can be turned off and $\rho$ can be set to a fixed value such as 0 to have a robust system performance around the steady state.

\section{$B$. Online tuning strategy for the shape of the SMF on the zT2-FPI controller (ST-zT2-FPI)}

In this subsection, we will present a simple mechanism to tune the shape of the SMF on the zT2-FPI controller in an online manner. As it has been shown in the derivations in Subsection (4.2), tuning the shape of the SMF can substantially improve the transient and disturbance rejection performances while preserving the robustness of an IT2-FPI controller. We will propose a heuristic tuning strategy, which is similar to the one presented in the previous subsection since the effect of $\gamma$ on the system response is similar to the effect of $\rho$ ), to generate an appropriate $\gamma$ in an online manner :

- At region 1, the value of $\gamma$ should be decreased in time to speed the system response sufficiently as:

$$
\gamma=\varphi * \sigma_{2}(e)
$$

Here, the value of " $\varphi$ " is taken to be " 1 " for all the regions except the first region. Since the error value possesses its extreme value at the first region, the value of " $\varphi$ " can take values like 0.4 or 0.5 so as to prevent the excessive acceleration of the system response which may cause possible overshoots.

- At region 2, $\gamma$ should be increased sufficiently to decrease the damping of control system as follows:

$$
\gamma=\sigma_{1}(e)
$$

- At region 3, the damping of control system should be decreased in time to accelerate the system response sufficiently. Thus, the tuning parameter is tuned as 


$$
\gamma=\sigma_{1}(e)
$$

- At region 4, $\gamma$ should be increased sufficiently to reduce the undershoot as follows:

$$
\gamma=\sigma_{2}(e)
$$

If the error $(e)$ and change of error $(\dot{e})$ has entered and remained within a specified error band, such as $2 \%$, then the tuning mechanism can be turned off and $\gamma$ can be set to a fixed value such as 0.4 or 0.5 to have a robust system performance and a fast disturbance rejection response around the steady state.

\section{The zT2 FPI Controller Design Strategy}

In this section, we will present the design strategy employed for zT2 FPI controllers. Since a systematic design even for IT2-FLCs is still a challenging problem, we will design the zT2-FPI controller by employing an evolutionary algorithm called Big Bang-Big Crunch (BB-BC) optimization. Thus, we will start by presenting briefly the BB-BC method and then we will present the design strategy employed for zT2 FPI controllers. It should be noted that, other evolutionary algorithms can also be employed to design the controllers. However, this does not affect the effectiveness of the presented results in this paper.

\section{A. A brief overview on BB-BC Optimization}

Erol and Eksin [30] proposed a new evolutionary algorithm named BB-BC. The working principle of this method can be explained as the transformation of a convergent solution to a chaotic state and then back to a single tentative solution point. BB-BC method consists of two main steps namely "Big Bang" and Big Crunch". In the BB-BC algorithm, the first step is the "Big Bang" phase where candidate solutions are randomly distributed over the search space. After the "Big Bang" phase, a contraction operator is applied such as the "Big Crunch" phase to form a center or a representative point for further "Big Bang" operations [30]. The contraction operator computes the center of mass which is defined as:

$$
x_{c}=\sum_{k=1}^{T} \frac{1}{f^{k}} x^{k} / \sum_{k=1}^{T} \frac{1}{f^{k}}
$$

where $x_{c}$ is the position of the center of mass, $f^{k}$ is the cost value of the $k^{\text {th }}$ candidate $x^{k}$, and $T$ is the population size. Then, in the next "Big Bang" phase, the new candidates are calculated as:

$$
x^{\text {new }}=x_{c}+\left(r \omega\left(x_{\max }-x_{\text {min }}\right)\right) / l
$$

where $r$ is a random number; $\omega$ is a parameter limiting the size of the search space, $x_{\max }$ and $x_{\min }$ are the upper and lower limits; and $l$ is the iteration step [30].

\section{B. The BB-BC optimization of the zT2 FPI Controller Structure}

In this subsection, we will present the application of BB-BC optimization to design the parameters of the antecedent MF of the zT2 FPI controller. Since T2-FLS is a generalization of T1-FLS, we will first design a baseline T1-FPI and then extend the controller to design the IT2-FPI and zT2-FPI controllers.

We will optimize the T1-FPI controller presented in Equation (2) to minimize the Integral Absolute Error (IAE) value via the BB-BC optimization. Thus, the optimization variables for the BB-BC algorithm are defined as $x_{T 1-F P I}=$ $\left(c_{\dot{e} 1}, c_{e 1}, K_{P}, K_{I}\right)$. While in the optimization of the IT2-FPI controller, we will employ $\alpha_{\dot{e}}=\alpha_{\dot{e}-1}=\alpha_{\dot{e} 1}$ and $\alpha_{e}=$ $\alpha_{e-1}=\alpha_{e 1}$ so that the properties of the antecedent MFs of IT2-FPI controller structure presented in Equation (4) are satisfied and accordingly only the heights $\left(\alpha_{\dot{e}}, \alpha_{e}\right)$ of the lower antecedent MFs are optimized to minimize the IAE value, while the cores $\left(c_{\dot{e} 1}, c_{e 1}\right)$ and consequent parameters $\left(K_{P}, K_{I}\right)$ are fixed and set to the same values of the Optimized T1-FPI (OT1-FPI) structure. Thus, the optimization variables for the BB-BC algorithm are defined as $x_{I T 2-F P I}=\left(\alpha_{\dot{e}}, \alpha_{e}\right)$.

As it has been shown in Subsection (3.2), the zT2-FPI can be constructed as a collection of IT2-FPI controllers. Thus, we will set the cores of the MFs and the consequent parameters of zT2-FPI to the same values of ones of the IT2-FPI. In the design of the FT-zT2-FPI $(\gamma=0)$, we will set the MF parameters of Optimized IT2-FPI (OIT2-FPI) as the lower bounds of the antecedent MFs of the FT-zT2-FPI (presented in Equations (73) and (74)), i.e. $\alpha_{\dot{e}_{0}}^{z_{1}}=\alpha_{\dot{e}}$ and $\alpha_{\dot{e}_{0}}^{z_{1}}=\alpha_{e}$. The parameters for MFs of the FT-zT2-FPI $\left(\alpha_{\dot{e}_{\text {new }}}^{z_{1}}, \alpha_{e_{\text {new }}}^{z_{1}}\right)$ will be online calculated as presented in Section (5.1). In the design of the ST-zT2-FPI $(0<\gamma \leq 1)$, we will set the MF parameters of OIT2-FPI as heights of the lower MFs of the ST-zT2-FPI associated with the zSlices $z_{1}$, i.e. $\alpha_{\dot{e}}^{z_{1}}=\alpha_{\dot{e}}$ and $\alpha_{e}^{z_{1}}=\alpha_{e}$. The parameters of the MFs associated with the zSlices $z_{2}$ $\left(\alpha_{\dot{e}}^{z_{2}}, \alpha_{e}^{z_{2}}\right)$ will be online obtained via the tuning mechanism presented in Section 5.2. The pseudo-code of the controllers design method is given in Table I.

\section{SIMULATION EXPERIMENTS AND RESULTS}

In this section, the performance of the proposed online FTzT2-FPI and ST-zT2-FPI are compared with the OT1-FPI and the OIT2-FPI controllers on two systems which have nonminimum phase and nonlinear dynamics, respectively. In this context, we will examine the transient state response, disturbance rejection performances and the robustness against nonlinear dynamics, parameter uncertainties and noise of the controllers. Thus, three performance measures are considered which are the settling time $\left(T_{s}\right)$, and the overshoot (OS\%) while the third performance measure is the IAE value. Throughout the studies, two MFs are used to characterize each input domain $(e, \dot{e})$ of the fuzzy structures where the cores of the MFs are fixed to $c_{\dot{e} 1}=c_{e 1}=1$. The design of the T1-FPI, IT2-FPI and zT2-FPI controllers have been accomplished as it has been stated in Section 5. In the simulation studies, all controllers are implemented as the discrete-time versions obtained with the bilinear transform with the sampling time $T_{s}=0.1 \mathrm{~s}$. The simulations were performed on a personal computer with an Intel Pentium Dual Core T2370 $1.73 \mathrm{GHz}$ processor, 2.99 GB RAM, and software package MATLAB/Simulink 7.4.0.

\section{A. Simulation Results on System I}

Consider the non-minimum phase process given by Astrom and Hagglund [28],

$$
G(s)=\frac{K(1-\tau s)}{(s+1)^{3}}
$$


where $K$ is the gain and $\tau$ determines the position of the right half plane zero and the nominal values are $K=1$ and $\tau=1$. The obtained optimal parameter sets for the controllers are tabulated in Table II.

The unit step responses and disturbance rejection performances of the fuzzy control systems are investigated for the nominal parameter set $K=1, \tau=1$ (Nominal System) and for three perturbed parameter sets which are $K=1.2, \tau=$ 1.6 (Perturbed System-1), $K=0.8, \tau=2.5 \quad$ (Perturbed System-2) and $K=1.5, \tau=0.5$ (Perturbed System-3) to examine their robustness against parameter variations. The output and input disturbance with the magnitudes of " 0.2 " have been applied in $50^{\text {th }}$ and $100^{\text {th }}$ seconds, respectively. The control performances of the fuzzy control systems are illustrated in Fig. 9. The transient state performance measures are given Table III while the output $\left(I A E_{d y}\right)$ and input $\left(I A E_{d u}\right)$ disturbance rejection performance measures are tabulated in Table IV.

As it can be clearly seen in Table III and Table IV, the OIT2-FPI is more robust against parameter partitions in comparison to its type- 1 counterpart. The OIT2-FPI system reduced the OS\% and oscillations by reducing the speed of the closed system response and accordingly has higher $T_{s}$ and IAE values and resulted with poor disturbance rejection performances. However, the proposed zT2-FPI structures enhanced both the transient state and disturbance rejection performances of all systems. For instance, if we examine the results for Perturbed System-1 (presented in Fig.9a), when compared to the OT1-FPI, the ST-zT2-FPI reduces the overshoot by about $24 \%(=\mathrm{abs}(26 \%-34 \%) / 34 \%)$; it also decreases the settling time by about $26 \%$ and the total IAE value by about $9 \%$. Note that, the OS\% and IAE values of the FT-zT2-FPI are quite close to the ST-zT2-FPI ones. However, in comparison to the ST-zT2-FPI structure, the FT-zT2-FPI inherits a relatively higher undershoot and oscillations. Moreover, when we examine the disturbance rejection performances from Table IV, it can be clearly seen that when compared to the OIT2-FPI controller, the zT2-FPI structures have better output $\left(I A E_{d y}\right)$ and input $\left(I A E_{d u}\right)$ disturbance rejection performance values. However, the best performance values are obtained by the ST-zT2-FPI which decreased $I A E_{d y}$ value by about $34 \%$ and $I A E_{d u}$ value by about $40 \%$ in comparison to OIT2-FPI. Similar comments can be made for the perturbed system responses. It can be concluded that the transient and disturbance rejection performances of the STzT2-FPI is better than the OT1-FPI, FT-zT2-FPI and OIT2FPI controllers while it is robust against parameter variations and disturbances.

In order to demonstrate the convergence of the tuning parameters of the FT-zT2-FPI and ST-zT2-FPI, we will show in Fig. 9 the change of the tuning parameters $(\gamma, \rho)$ for the presented parameter settings. As can be seen in Fig. 9, the value of the tuning parameters relatively increases to accelerate the system response sufficiently while preventing possible overshoot as the system response approaches the setpoint. The same performances of $(\gamma, \rho)$ follows for all the following experiments, however due to the space constraints, we will not show the change of the tuning parameter in the rest of the paper.

\section{B. Simulation Results on System II}

Consider the nonlinear system given by Mudi and Pal [29] as

$$
u(t-L)=\frac{d^{2} y(t)}{d t^{2}}+\frac{d y(t)}{d t}+k y(t)^{2}
$$

where $L$ is the time delay and $k$ is the gain. Moreover, we will assume that the output inherits band limited white noise to examine also the robustness of the controllers against noise. The obtained optimal parameter sets of the fuzzy controllers for $L=0.4, k=0.25$ (Nominal System) are given in Table II.

The step responses and disturbance rejection performances of the controllers are investigated for the nominal parameter set and for the perturbed parameter set which is $L=0.8, k=$ 0.15 (Perturbed System) to examine their robustness against parameter uncertainties. The system responses are illustrated in Fig. 10 while the performance measures are given in Table $\mathrm{V}$. Here, the output and input disturbance with the magnitudes of " 0.2 " have been applied in $50^{\text {th }}$ and $100^{\text {th }}$ seconds, respectively.

The OIT2-FPI structure was able to reduce the overshoot and provide robustness against parameter variations, noise and parameter variations in comparison to its type-1 while reducing the system response time which resulted as poor disturbance rejection performances. On the other hand, both zT2-FPI structures provide satisfactory transient state and disturbance rejection performances in the presence of noise as has been shown in Fig.10. For the nominal system, the FTzT2-FPI and the ST-zT2-FPI structures have almost identical performance values but the FT-zT2-FPI is slightly better as given in Table V. While in the perturbed system responses, when compared to OT1-FPI, the ST-zT2-FPI reduces the overshoot by about $34 \%$; it also decreases the settling time by about $39 \%$ and reduced the total IAE value by about $18 \%$. Although the FT-zT2-FPI has an identical OS with the STzT2-FPI, it has a bigger settling time and IAE values by about $22 \%$ and $15 \%$, respectively in comparison to the ST-zT2-FPI. Moreover, the system response of the FT-zT2-FPI inherits a fairly large undershoot and oscillations which are avoided by the ST-zT2-FPI. Moreover, when we examine the disturbance rejection performance measures given in Table $\mathrm{V}$, it can be clearly seen that when compared to the OIT2-FPI structure, the FT-zT2-FPI enhances the both $I A E_{d y}$ and $I A E_{d u}$ values by about $64 \%$ for the perturbed system. Note that, although the disturbance rejection performance is satisfactory in the sense of the $I A E_{d y}$ and $I A E_{d u}$ values, the system response is poor since it inherits a large overshoot and oscillations. It can be concluded that the FT-zT2-FPI provided a robust control performance against nonlinear dynamics, noise and disturbances while providing better the performance measures than the OT1-FPI and OIT2-FPI as tabulated in Table V.

\section{General Comments on the zT2-FPI control system performance}

As we have illustrated in the simulation studies, online tuning the size and shape of the SMFs of the zT2-FPI can enhance the system performance in comparison to its type-1 and type- 2 counterparts. In the first proposed FT-zT2-FPI (since $\gamma=0$ it is in fact an IT2-FPI with an online FOU tuning mechanism) structure, we have shown that tuning the size of the FOUs of the IT2-FPI might not be an effective way 
since the system response inherits oscillations and undershoots similar to its type-1 counterpart in presence of noise, parameter variations, disturbances and nonlinear dynamics. While in the presented results of the proposed ST-zT2-FPI confirm that tuning the shape of the SMFs with fixed FOU size provides robustness against noise, parameter variations and nonlinear dynamics. There are improvements both in the transient state and disturbance rejection performances such as reducing the overshoot and settling time and eliminating undershoots and oscillations. Thus, in the real-time studies of the mobile robot where the possibility of noise and unmodelled nonlinear dynamics are high (which will be presented in the following section) only the ST-zT2-FPI structure will be employed and abbreviated as "EzT2-FPI".

Moreover, to establish if the proposed zT2-FPI more complex structures will cause drastic impact on the controllers real-time response, we have investigated the computation time needed by the zT2-FPI, IT2-FPI and T1-FPI structures to map an input to an output for all possible combinations of the input values in their corresponding universe of discourses, i.e., $e \in[-$ $1,+1]$ and $\dot{e} \in[-1,+1]$. It was found that the average computation times of the T1-FPI, IT2-FPI and zT2-FPI structures are $0.12 \mathrm{~ms}, 0.29 \mathrm{~ms}$ and $0.42 \mathrm{~ms}$ respectively, where the obtained maximum computation time values are $13.62 \mathrm{~ms}$, $19.57 \mathrm{~ms}$ and $22.62 \mathrm{~ms}$, respectively. Although there is some increase in the average computation time of the zT2-FPI in comparison to the T1-FPI and IT2-FPI, the obtained maximum computation times of the IT2-FPI and zT2-FPI are quite close. Hence, the real-time control application of the zT2-FPI is feasible for systems with relatively small sampling periods and it will be shown later that the proposed zT2-FPI produces a superior transient state and disturbance rejection performances in comparison to the T1-FPI and IT2-FPI.

\section{REAL-WORLD EXPERIMENTS AND RESULTS}

In this section, we will compare the performance of the OT1-FPI, OIT2-FPI and EzT2-FPI (ST-zT2-FPI) controllers in a cascade structure to solve the path tracking control problem of a real-world mobile robot. Thus, we will start by presenting kinematic motion equations of the mobile robot and then present the proposed cascade structure for the tracking control problem of the mobile robots. Finally, the real-time control performance of the presented type- 1 and type- 2 structures are examined.

\section{A. The Kinematic Motion Equations of the Mobile Robot}

The schematic diagram of the Pioneer 3-DX mobile robot's architecture is illustrated in Fig. 11a. The kinematic motion equations of the mobile robot have a nonintegrable constraint with the assumption that the robot cannot slip in a lateral direction in the form as:

$$
A(q) \dot{q}=\dot{x} \sin \theta-\dot{y} \cos \theta
$$

where $A(q)$ is the constraint matrix defined over the state vector $q(t)=[x(t) y(t) \theta(t)]^{T}$. The first order kinematics model is in the following equation:

$$
\dot{q}=s(q)\left[\begin{array}{ll}
v & w
\end{array}\right]^{T}
$$

where

$$
s(q)=\left[\begin{array}{cc}
\cos \theta & 0 \\
\sin \theta & 0 \\
0 & 1
\end{array}\right]
$$

and $v(t)$ and $w(t)$ are the linear and angular velocities of the mobile robot. Moreover, the bounded velocity and acceleration constraints are considered [31]. To control the system given in Equation (89), the reference trajectory should also be described as:

$$
\dot{q}_{r}=\left[\begin{array}{cc}
\cos \theta_{r} & 0 \\
\sin \theta_{r} & 0 \\
0 & 1
\end{array}\right] u_{r}
$$

where $q_{r}(t)=\left[x_{r}(t) y_{r}(t) \theta_{r}(t)\right]^{T}$ is the reference state vector and $u_{r}(t)=\left[\begin{array}{lll}v_{r}(t) & w_{r}(t)\end{array}\right]^{T}$ is the reference control signal. Then, an error state can be defined as follows:

$$
\dot{q}_{e}=\left[\begin{array}{l}
e_{x} \\
e_{y} \\
e_{\theta}
\end{array}\right]=\left[\begin{array}{ccc}
\cos \theta & \sin \theta & 0 \\
-\sin \theta & \cos \theta & 0 \\
0 & 0 & 1
\end{array}\right]\left[\begin{array}{l}
x_{r}-x \\
y_{r}-y \\
\theta_{r}-\theta
\end{array}\right]
$$

Consequently, the tracking control problem is converted into a regulation problem [32].

\section{B. The Proposed Cascade Control Structure for the Mobile Robot Tracking Control}

In this subsection, we will present a cascade structure to solve the path tracking control problem of the mobile robot. In this study, we will consider the linear velocity $(v(t))$ to be constant while the angular velocity of the mobile robot as the manipulated variable $(w(t))$. Consequently, the mobile robot model should have a single input and three controlled variables, it has been reported that the system performance is also satisfactory when two controlled variables $(\theta(t)$ and $y(t))$ are considered since $x(t)$ can be defined as a function of $\theta(t)$ and $y(t))$ [33], [34]. The employed overall cascade architecture is illustrated in Fig. 12. The implemented cascade structure consists of three main blocks which are the transformation block, the outer and the inner controller. In the transformation mechanism, the robot pose $(x(t), y(t), \theta(t))$ and the desired reference trajectory is used to compute the local error signal on y- axis via:

$$
e_{y}=\cos \theta\left(y_{r}-y\right)-\sin \theta\left(x_{r}-x\right)
$$

This error signal is then fed as the input to the outer controller to generate the reference angle $\theta_{\text {ref }}(t)$ which will correspond to the necessary heading angle of the inner controller to track the trajectory.

In the real time applications, the inner controller is chosen as PI controller and its parameters are optimized to minimize the IAE performance index via the BB-BC optimization algorithm. Here, the linear speed of the mobile robot is set as $v(t)=200 \mathrm{~cm} / \mathrm{s}$ and the starting point of the mobile robot was the center on the origin of the coordinate system $(x=0 \mathrm{~m}$, $y=0 \mathrm{~m})$ with the initial heading of $\theta=0$. The optimum values of parameters are found as $K_{P}=3.01$ and $K_{\Gamma}=0.24$ for the reference steering angle $\theta_{\text {ref }}=\pi / 2$. The presented OT1-FPI, OIT2-FPI and EzT2-FPI are implemented as the outer controller for performance comparison, respectively. The design procedure presented in Section 5 has been employed for a $y$-axis step reference with a value of $500 \mathrm{~cm}$. The obtained values of OT1-FPI are $K_{P}=0.25, K_{I}=0.9, c_{\dot{e} 1}=$ $4.5, c_{e 1}=2$ and for the OIT2-FPI are $\alpha_{\dot{e}}=0.42, \alpha_{e}=0.64$. In the design of EzT2-PI, the defined parameter $(\gamma)$ is online tuned while the other parameters are set as given in Section 5. 


\section{Real-Time Closed Loop Control Performance Analysis}

In this subsection, we will present real world experiments using the PIONEER 3-DX mobile robot (shown in Fig. 11b) to evaluate the proposed EzT2-FPI structure. The pose of the mobile robots $(x(t), y(t), \theta(t))$ has been computed at each sampling time $\left(t_{s}=0.1 \mathrm{~s}\right)$ using the odometry system. It is well known that odometry is a technique which has an accumulative error which implies the need to update the estimation from data provided from an external sensor system at a predetermined sampling period [31], [32]. This issue is separate from the control problem, and has not been addressed in the paper. The experimental environment of the mobile robot is shown in Fig. 11c. It should be noted that the presented trajectory in Fig. 11c is just for visualization of the reference trajectory. The reference trajectory is predefined and implemented in the reference path mechanism which is illustrated in Fig. 12.

In the real-time control studies, different analyses are presented to investigate the control performances of the OT1FPI, OIT2-FPI and EzT2-FPI structures. Thus, we will examine the transient state response and disturbance rejection performances of the controllers for various operating points and investigate robustness against parameter uncertainties, nonlinear dynamics, disturbances and noises. To make a fair comparison, three performance measures are considered which are $T_{s}, \% O S$ and

$$
\begin{aligned}
& I A E_{y}=\int\left|y_{r}-y\right| \text { for } \mathrm{y}-\text { axis reference values } \\
& I A E_{x}=\int\left|x_{r}-x\right| \text { for } \mathrm{x}-\text { axis reference values } \\
& \text { 1) Y-Axis Trajectory Control Performance Analysis }
\end{aligned}
$$

\section{1) Y-Axis Trajectory Control Performance Analysis}

This section presents an experimental comparison of the cascade structures for a constant $y$-axis reference value. We will examine the control performance of the fuzzy PI controllers in three cases. We will first start the control performance of the controllers at the operating point (Case-1: $\operatorname{yr}(\mathrm{t})=500 \mathrm{~cm}, \mathrm{v}(\mathrm{t})=200 \mathrm{~m} / \mathrm{s})$ at which the controllers were designed. Then, at the same operating point, we will increase the constant linear velocity $15 \% \quad($ Case -2 : $\operatorname{yr}(\mathrm{t})=500 \mathrm{~cm}$, $\mathrm{v}(\mathrm{t})=230 \mathrm{~m} / \mathrm{s})$ to see how the controllers are robust against parameter uncertainties. Moreover, the robustness of the controllers against nonlinear dynamics is investigated by defining another $y$-axis reference trajectory (Case-3: $\operatorname{yr}(\mathrm{t})=300 \mathrm{~cm}, \mathrm{v}(\mathrm{t})=200 \mathrm{~m} / \mathrm{s})$.

The tracking results on the $\mathrm{x}-\mathrm{y}$ axis for all three cases are shown in Fig. 13 while performance measures are given in Table VI. The experimental results of the EzT2-FPI structure show that the mobile robot converges to the reference trajectory in short time when compared to the OT1-FPI and OIT2-FPI counterparts. For instance, if we examine the results for Case-1, when compared to OT1-FPI, the EzT2-FPI structure reduces the overshoot by about 61\%; it also decreases the settling time by about $43 \%$ and it reduces the total IAEy value by about $27 \%$. The transient performance measures of the OIT2-FPI are also satisfactory. OIT2-FPI enhanced both the overshoot and settling time but with a relatively high IAEy performance value which is almost 1.3 times bigger than the EzT2-FPI ones. Similar comments can be made for Case-2. Moreover, if we examine the results of
Case-3 it can be clearly seen that the T1-FPI system response is oscillating while both the OT2-FPI and EzT2-FPI were able to converge to reference value. However, as tabulated in Table VI, EzT2-FPI structure resulted in the lowest Ts and IAEy performance values when compared to the OIT2-FPI counterpart. It can be concluded that, the EzT2-FPI structure preservers the robustness against parameter uncertainties and nonlinear dynamics while enhancing the transient state performance in comparison to the type- 1 and type- 2 fuzzy counterparts.

\section{2) X-Axis Trajectory Control Performance Analysis}

We have tested the performances of the controllers also for $\mathrm{x}$-axis reference trajectory with a the initial pose of $(x(t), y(t), \theta(t))=(0 \mathrm{~m}, 0 \mathrm{~m}, 3 \pi / 2)$. Since the controllers were designed for $\mathrm{y}$ axis trajectory, this could be a good way to investigate the transient performances and how robust the controllers are against nonlinear dynamics and parameter variations. Thus, we will examine the control performance of the fuzzy PI controllers in two operating points which are $x_{r}(t)=500 \mathrm{~cm}, \quad v(t)=200 \mathrm{~m} / \mathrm{s} \quad($ Case- 4$)$ and $x_{r}(t)=500 \mathrm{~cm}$, $v(t)=230 \mathrm{~m} / \mathrm{s}$ (Case-5). In Fig.14, the transient convergence of the mobile robot to $\mathrm{x}$-axis reference trajectory is given for the two examined cases. The EzT2-FPI provides a better control performance than the type- 1 and type- 2 fuzzy structures as given in Table VII. For all cases, only the presented the OIT2FPI and the EzT2-FPI were able to converge to the desired value. However, the EzT2-FPI structure was able to accelerate the system response and enhance the $I A E_{x}$ value in comparison to the IT2-FPI while the OT1-FPI was not able to handle different operating conditions. The results confirm that the EzT2-FPI structure has the ability to enhance the transient state and be more robust against nonlinear dynamics and parameter uncertainties at various operating points in comparison to the other controllers.

3) Disturbance Rejection Performance Analysis

This subsection examines the disturbance rejection performances of the fuzzy controllers. We will examine the robustness against disturbances by presenting both the input and output disturbances. The robustness against disturbances will be examined for the nominal velocity $(v(t)=200 \mathrm{~cm} / \mathrm{s})$ and then we will increase the velocity by about $15 \%$ $(v(t)=230 \mathrm{~cm} / \mathrm{s})$ to investigate the robustness in the presence of parameter uncertainties. The mobile robot is in steady state at the operating point $(y(t)=0, \theta(t)=0, w(t)=0)$.

At first, a step input disturbance $\left(d_{w}(t)\right)$ with a magnitude of " 0.5 " has been applied the mobile robot. The input disturbance performance for the nominal (Case-6: $v(t)=200 \mathrm{~cm} / \mathrm{s}$ ) and perturbed (Case-7: $v(t)=230 \mathrm{~cm} / \mathrm{s}$ ) are presented in Fig.15. If we examine the performance results given in Table VIII, it is clear that when compared to IT2-FPI, the EzT2-FPI structure reduces $I A E_{d u}$ value by about $46 \%$ for the nominal linear velocity while for the perturbed linear velocity, the EzT2-FPI improves the performance by about $12 \%$. It should be noted that the $I A E_{d u}$ value of the OT1-FPI has the lower value for the nominal velocity while a higher value for the perturbed velocity in comparison with the EzT2FPI controller's values. The results confirm that OT1-FPI is not robust in various operating conditions and nonlinearities while the EzT2-FPI structure is more robust against input disturbances and parameter variations and is capable to 
enhance the disturbance rejection performance of OIT2-FPI structure.

Secondly, a step output disturbance $\left(d_{y}(t)\right)$ with a magnitude of " 200 " has been applied the mobile robot. The output disturbance rejection performances of the fuzzy controllers for the nominal (Case-8: $v(t)=200 \mathrm{~cm} / \mathrm{s}$ ) and perturbed (Case-9: $v(t)=230 \mathrm{~cm} / \mathrm{s})$ linear velocity are presented in Fig.16. The performance measures of the fuzzy controllers are tabulated in Table VIII. The system response of the OT1FPI structure is oscillating for the nominal speed while resulted with a satisfactory disturbance rejection performance for the perturbed speed. This confirms that OT1-FPI is not robust in different operating conditions. Nevertheless, for both linear speeds the OIT2-FPI and EzT2-FPI were able to obtain a non-oscillating system response. In comparison to OIT2-FPI, the EzT2-FPI structure was able to accelerate the disturbance rejection performance significantly and consequently reduced $I A E_{d u}$ value to about $15 \%$ for the nominal velocity while for the perturbed velocity by about $23 \%$.

The performance measures of the disturbance rejection studies confirm that the EzT2-FPI is more capable of handling disturbances and is more robust against nonlinear dynamics and uncertainties while providing a fast and satisfactory disturbance rejection performance when compared to IT2-FPI structure.

\section{CONCLUSIONS AND FutURE WORK}

It has been shown in various works that the IT2-FLCs might be able to handle high levels of uncertainties since it they have a smoother control surface around the steady state. Thus, it had been deduced that IT2-FPI controllers are potentially more robust; however, the settling time may increase and disturbance rejection performance may degrade in comparison with its type- 1 counterparts. Thus, to combine the features of the T1-FPI and IT2-FPI controllers (speed and robustness), we developed a general type-2 fuzzy PI controller which provided an acceptable trade-off between the robust control performance of the IT2-FPI and the acceptable transient and disturbance rejection performance of the T1-FPI controllers. It should be stressed that the paper focused on the case followed in conventional or self-tuning fuzzy controller design strategies where the aim is to decrease the integral action sufficiently around the steady state to have robust system performance against noises and parameter variations.

In this paper, we proposed a novel zT2-FPI structure where the SMFs of the antecedent T2-FSs are adjusted in an on-line manner. We first presented the internal structure of the proposed zT2-FPI controller which is in fact a series of slightly modified IT2-FPI controllers due to the zSlices theory. We proposed a simple method to design zT2-FPI controllers by a single design parameter which determines the shape of the SMFs of the antecedent T2-FSs. We provided theoretical explanations showing how the size and shape of the SMFs affects the controller performance from a mathematically point of view for the first time in literature. We have presented analysis showing that increasing the size of the FOUs of the zT2-FPI can potentially make the system more robust against parameter variations and improve the transient state performance such as reducing the overshoot and oscillations on the cost of a slower system response and disturbance rejection performance aspects when compared to the type-1 counterparts. We also provided theoretical analysis showing that tuning the shape of the SMFs of the zT2-FPI controllers is an efficient way to provide an acceptable trade-off between the transient state and disturbance rejection performance of the zT2-FPI controller while preserving the robustness against parameter variations, noise and uncertainties. Based on our observations of the tuning parameters $\rho$ (which is used to tune the size of the FOU) and $\gamma$ (which is used to design the shape of the SMFs) on the system response; we proposed two heuristic tuning mechanism to shape the system response to obtain an efficient and appropriate control signal that will be able achieve a desired transient state response and an efficient disturbance rejection performance while preserving the robustness of IT2-FPI controllers. Moreover, we presented a BB-BC optimization based zT2-FPI design strategy.

We presented several simulation studies to validate the proposed approaches where the EzT2-FPI structures were compared with an OT1-FPI and OIT2-FPI structures with respect to defined performance measures. We have illustrated that tuning the shape of the SMF of the zT2-FPI structure is a more efficient control strategy instead of tuning the size of FOU of the zT2-FPI (actually a self-tuning IT2-FPI) structure. We also illustrated that the proposed controller has relatively low computational cost which makes it feasible for real-time control applications with relatively small sampling periods since zT2-FPI employed the zSlices theory and tuned by a single parameter. We presented also real time control studies to evaluate the controllers on the real-time control performance of the PIONEER 3-DX mobile robot which inherit large amounts of nonlinearities and uncertainties caused by the internal dynamics and/or feedback sensors of the controlled system. The presented comparative experiments support the effectiveness of the proposed EzT2-FPI design approach. The experimental control performance results confirmed that the proposed EzT2-FPI structure can enhance the transient state and disturbance rejection control performances and it is also more robust to nonlinear dynamics, disturbances, noise and uncertainties when compared to the OT1-FPI and OIT2-FPI controllers for the handled benchmark systems.

It can be concluded that the proposed EzT2-FPI structure gives the opportunity to enhance both the transient state performance and disturbance rejection performance while preserving the robustness against nonlinear dynamics, noises and parameter uncertainties in different operating points and conditions which is not possible with the type- 1 and interval type- 2 counterpart for certain class of systems.

For our future work, we aim to develop and design zT2-FPI controllers having more than two zSlices $(Q>2)$ and extend the presented design approach. Moreover, we aim to focus on more sophisticated tuning mechanisms which might improve the control performance of the zT2-FPI control system.

\section{REFERENCES}

[1] D. Wu and W. W. Tan, "Interval type-2 fuzzy PI controllers: Why they are more robust," IEEE International Conference on Granular Computing, Silicon Valley, August 2010. 
[2] T. Kumbasar, I. Eksin, M. Guzelkaya and E. Yesil, "Type-2 fuzzy model based controller design for neutralization processes," ISA Transactions, vol. 51, no. 2, pp. $277-287,2012$.

[3] H. Hagras, "A Hierarchical Type-2 Fuzzy Logic Control Architecture for Autonomous Mobile Robots,” IEEE Trans. Fuzzy Syst., vol. 12, no. 4, pp. 524-539, 2004.

[4] D. Wu and W. W. Tan, "Genetic Learning and Performance Evaluation of Internal Type-2 Fuzzy Logic Controllers," Eng. Appl. Artif. Intel.Article, vol. 19, pp. 829-841, 2006.

[5] M. Galluzzo, B. Cosenza and A. Matharu, "Control of a Nonlinear Continuous Bioreactor with Bifurcation by a Type-2 Fuzzy Logic Controller," Computers \& Chemical Engineering, vol. 32, no.12, pp. 2986-2993, 2008

[6] J. S. Martínez, J. Mulot, F. Harel, D. Hissel, M. C. Péra, R. I. John and M. Amiet," Experimental validation of a type-2 fuzzy logic controller for energy management in hybrid electrical vehicles", Eng. Appl. Artif. Intel. Article in press, 2013.

[7] Q. Liang and J.M. Mendel, "Interval Type-2 Fuzzy Logic Systems: Theory and Design,” IEEE Trans. Fuzzy Syst., vol. 8, no.5, pp. 535-550, 2000.

[8] H. Mo, F.Y. Wang, M. Zhou, R. Li and Z. Xiao, "Footprint of uncertainty for type-2 fuzzy sets," Information Sciences, DOI:10.1016/j.ins.2014.02.092, 2014.

[9] L. Chengdong, J. Yi, G. Zhang, "On the monotonicity of interval type-2 fuzzy logic systems" IEEE Trans. Fuzzy Syst., DOI:10.1109/TFUZZ.2013.2286416, 2013

[10] O. Castillo and P. Melin, "A review on the design and optimization of interval type-2 fuzzy controllers," Applied Soft Computing, vol. 12, pp. 1267-1278, 2012.

[11] R. Martínez, O. Castillo, and L. T. Aguilar, "Optimization of interval type-2 fuzzy logic controllers for a perturbed autonomous wheeled mobile robot using genetic algorithms," Information Sciences, vol. 179, no: 13, pp. 2158-2174, 2009.

[12] S-K. Oh, H.-J. Jang, and W. Pedrycz, "A comparative experimental study of type-1/type-2 fuzzy cascade controller based on genetic algorithms and particle swarm optimization," Expert. Syst. Appl., vol. 38, no. 9, pp. 11217-11229, 2011.

[13] O. Castillo, R. Martínez, P. Melin, F. Valdez and J. Soria, "Comparative study of bio-inspired algorithms applied to the optimization of type-1 and type-2 fuzzy controllers for an autonomous mobile robot." Information Sciences, vol. 19, no. 2 pp. 19-38, 2012.

[14] D. Wu, "On the Fundamental Differences between Type-1 and Interval Type-2 Fuzzy Logic Controllers,” IEEE Trans. Fuzzy Syst., vol., 10, no.5, pp. 832- 848, 2012.

[15] X. Du and H. Ying, "Derivation and analysis of the analytical structures of the interval type-2 fuzzy-PI and PD controllers." IEEE Trans. Fuzzy Syst., vol. 18, no. 4, pp. 802-814, 2010.

[16] M. Nie and W. W. Tan, "Analytical structure and characteristics of symmetric Karnik-Mendel type-reduced interval type-2 fuzzy PI and PD controllers," IEEE Trans. Fuzzy Syst., vol., 20, no.3, pp. 416-430, 2012.

[17] S. Skogestad, "Tuning for smooth PID control with acceptable disturbance rejection, "Industrial \& Engineering Chemistry Research, vol. 45, no. 23, pp. 7817-7822, 2006.

[18] S. Alcántara, R. Vilanova, and C. Pedret. "PID control in terms of robustness/performance and servo/regulator trade-offs: A unifying approach to balanced autotuning." Journal of Process Control, vol. 23, no. 4, pp. 527-542, 2013.

[19] S. Coupland and R. John, "Geometric type-1 and type-2 fuzzy logic systems," IEEE Trans. Fuzzy Syst., vol. 15, no. 1, pp. 3-15, 2007.

[20] J. Mendel and F. Liu, "On new quasi-type-2 fuzzy logic systems," IEEE International World Congress on Computational Intelligence, Fuzz-2008, pp. 354-360, 2008.

[21] J. Mendel, F. Liu and D. Zhai, " $\alpha$-Plane Representation for Type-2 Fuzzy Sets: Theory and Applications," IEEE Trans. Fuzzy Syst., vol. 17, no. 5, pp. 1189-1207, 2009.

[22] S. Greenfield and F. Chiclana. "Defuzzification of the Discretised Generalised Type-2 Fuzzy Set: Experimental Evaluation." Information Sciences, vol. 244, pp.1-25, 2013.

[23] C. Wagner, and H. Hagras, "Toward general type-2 fuzzy logic systems based on zSlices", IEEE Trans. Fuzzy Syst., vol.18, no.4, pp. 637-660, 2010 .

[24] A. Bilgin, H. Hagras, A. Malibari, M. J. Alhaddad and D. Alghazzawi, "Towards a linear general type-2 fuzzy logic based approach for computing with words", Soft Comput ing, vol. 17, no:12, pp. 2203-2222, 2013 .
[25] M. Mizumoto, "Realization of PID controls by fuzzy control methods," Fuzzy Sets and Systems, vol. 70, pp. 171-182, 1995.

[26] H. Wu and J. Mendel, "Enhanced Karnik-Mendel Algorithms," IEEE Trans. Fuzzy Syst., vol. 17, no. 4, pp. 923-934, 2009.

[27] D. Zhai and J. Mendel, " Comment on "Toward General Type-2 Fuzzy Logic Systems Based on zSlices"," IEEE Trans. Fuzzy Syst., vol.20, no.5, pp. 996-997, 2012.

[28] K. J. Astrom and T. Hagglund," Advanced PID Control". Research Triangle Park, NC: ISA-The Instrumentation, Systems, and Automation Society, 2005.

[29] R. K. Mudi and N. R. Pal,. "A robust self-tuning scheme for PI- and PDtype fuzzy controllers," IEEE Trans. Fuzzy Syst., vol. 7, no.1, pp. 2-16, 1999.

[30] O. K. Erol and I. Eksin, "A New Optimization Method: Big Bang Big Crunch," Advances in Engineering Software, vol. 37, pp.106-111, 2006.

[31] G. Klančar, and I. Škrjanc, "Tracking-error model-based predictive control for mobile robots in real time." Robotics and Autonomous Systems, vol. 55, no: 6, pp. 460-469, 2007.

[32] D. Gu and H. Huosheng, "Receding horizon tracking control of wheeled mobile robots," IEEE Transactions on Control Systems Technology, vol. 14, no: 4, pp.743-749, 2006.

[33] J. E. Normey-Rico, I. Alcalá, J. Gómez-Ortega and E.F. Camacho, "Mobile robot path tracking using a robust PID controller," Control Engineering Practice, vol. 9, no.11, pp. 1209-1214, 2001.

[34] J. E. Normey-Rico, J. Gomez-Ortega and E.F. Camacho, "A Smith predictor based generalized predictive controller for mobile robot path tracking," Control Engineering Practice, vol. 7, pp. 729-740, 1999.

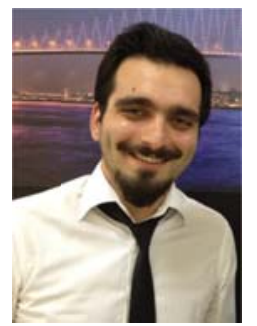

Tufan Kumbasar(M'13) received the B.Sc. and M.Sc. degrees in control engineering from Istanbul Technical University, Istanbul, Turkey, and the Ph.D. degree in control and automation engineering from the Istanbul Technical University. He is an Assistant Professor in the Control and Automation Engineering Department, Faculty of Electrical and Electronics Engineering, Istanbul Technical University. His major research interests are in computational intelligence, notably type-2 fuzzy systems, fuzzy logic, neural networks, evolutionary algorithms and control theory. $\mathrm{He}$ is also interested in process control, robotics and intelligent control. He has authored more than 200 papers in international journals, conferences and books.

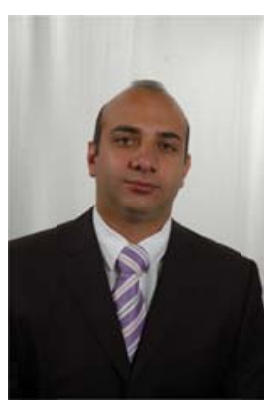

Hani Hagras (M'03-SM'05, F'13) received the B.Sc. and M.Sc. degrees in electrical engineering from Alexandria University, Alexandria, Egypt, and the Ph.D. degree in computer science from the University of Essex, Colchester, U.K. $\mathrm{He}$ is a Professor in the School of Computer Science and Electronic Engineering, Director of the Computational Intelligence Centre and the Head of the Fuzzy Systems Research Group in the University of Essex, UK. His major research interests are in computational intelligence, notably type-2 fuzzy systems, fuzzy logic, neural networks, genetic algorithms, and evolutionary computation. His research interests also include ambient intelligence, pervasive computing and intelligent buildings. He is also interested in embedded agents, robotics 
and intelligent control. He has authored more than 200 papers in international journals, conferences and books. He is a Fellow of the Institute of Electrical and Electronics Engineers (IEEE) and he is also a Fellow of the Institution of Engineering and Technology (IET (IEE). He was the Chair of IEEE Computational Intelligence Society (CIS) Senior Members Sub-Committee. His research has won numerous prestigious international awards where most recently he was awarded by the IEEE Computational Intelligence Society (CIS), the 2013 Outstanding Paper Award in the IEEE Transactions on Fuzzy Systems and he was also awarded the 2006 Outstanding Paper Award in the IEEE Transactions on Fuzzy Systems. He is an Associate Editor of the IEEE Transactions on Fuzzy Systems. He is also an Associate Editor of the International Journal of Robotics and Automation, the Journal of Cognitive Computation and the Journal of Ambient Computing and Intelligence. $\mathrm{He}$ is a member of the IEEE Computational Intelligence Society (CIS) Fuzzy Systems Technical Committee. Prof. Hagras chaired several international conferences where he served as the General CoChair of the 2007 IEEE International Conference on Fuzzy systems London, July 2007 and he served as the Chair of 2009, 2011, 2013 and 2014 IEEE Symposium on Intelligent Agents, he has also served as the Chair of 2011 IEEE Symposium on Advanced to Type-2 Fuzzy Logic Systems, Paris 2011. 


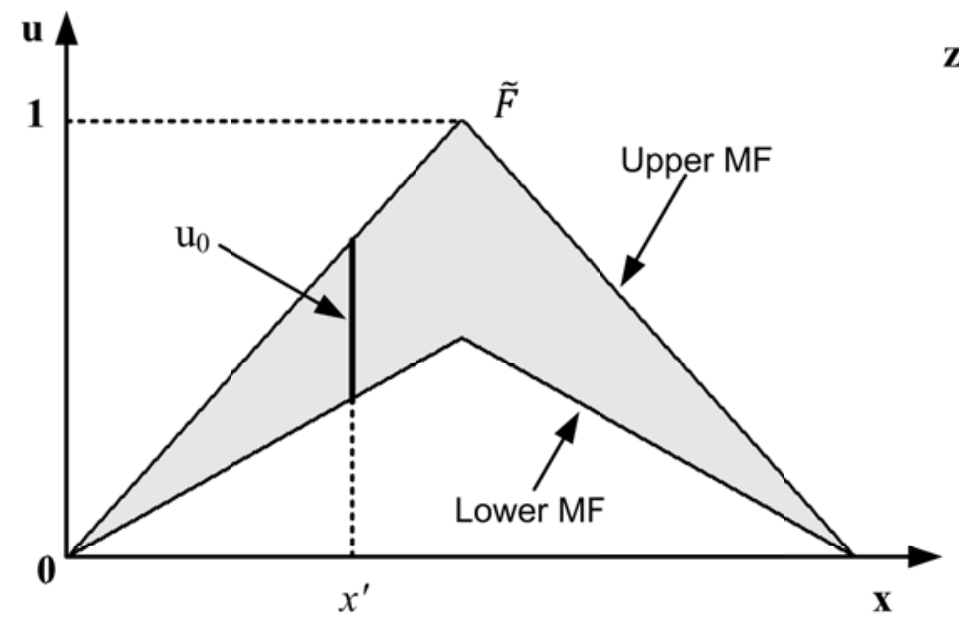

(a)

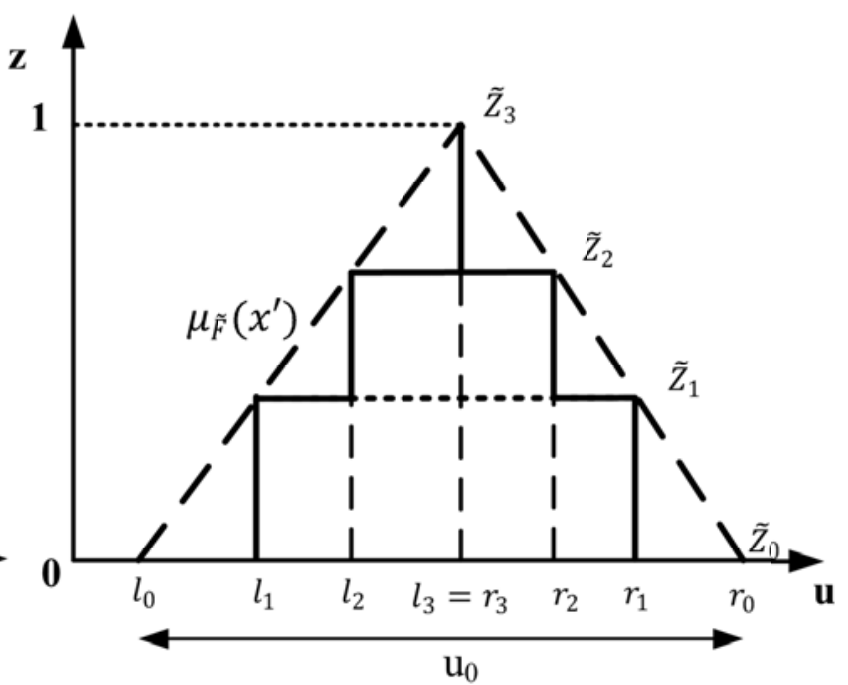

(b)

Fig. 1. Illustration of the (a) Front projection of a general T2-FS $(\tilde{F})$ in the $x$-u plane (b) The SMF at $x$ ' of the T2-FS (the dashed line) and its zSlices-based presentation (Solid line).

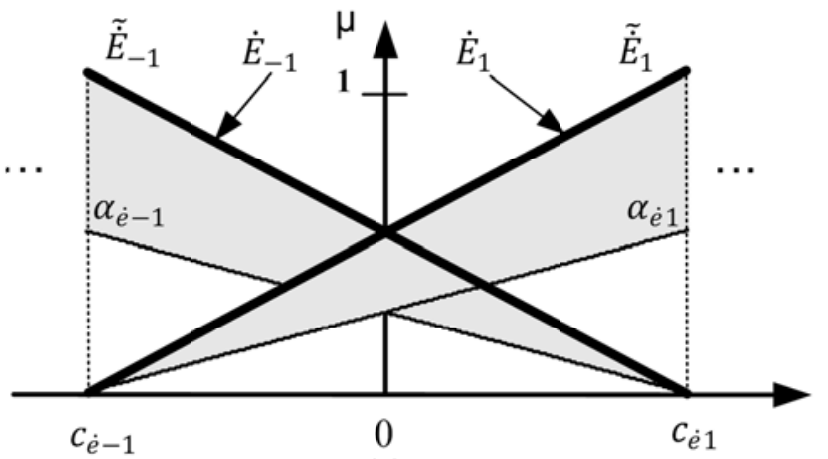

(a)

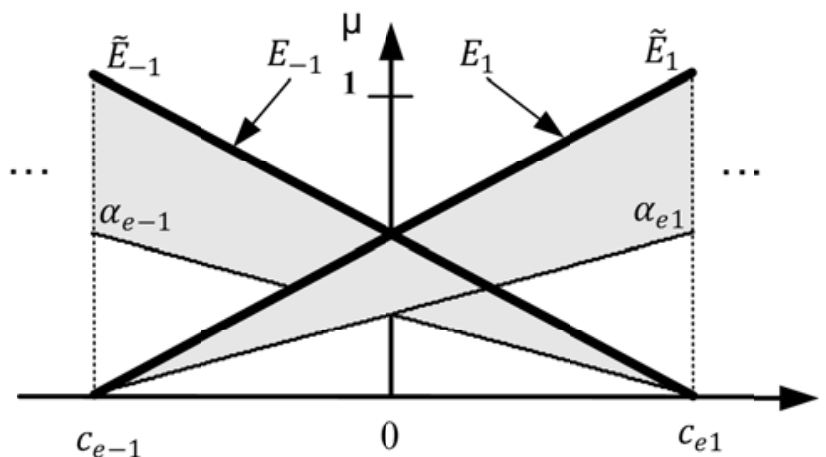

(b)

Fig. 2. The antecedent MFs around the origin for (a) the input e (b) the input e.

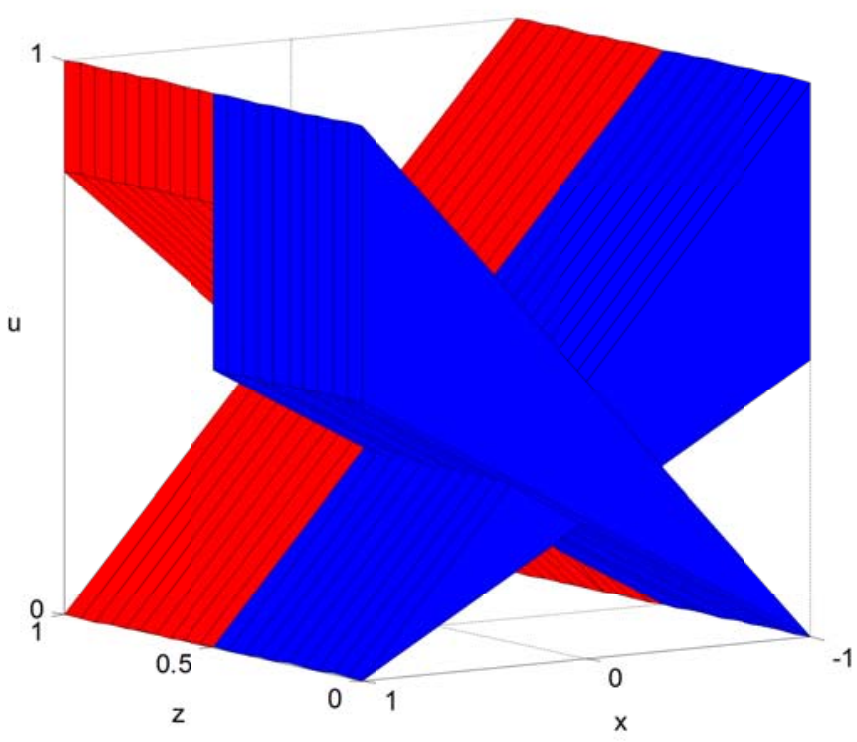

(a)

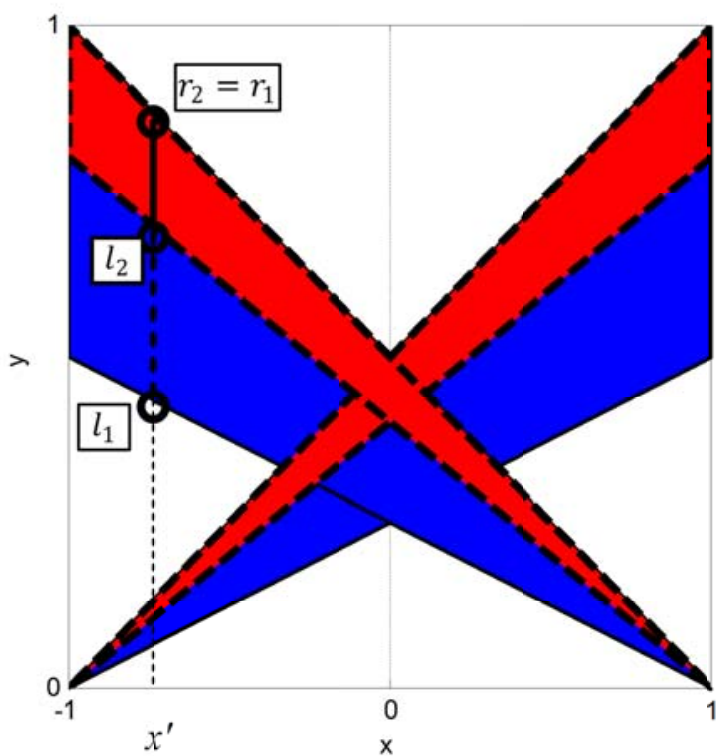

(b)

Fig. 3. Illustration of the zT2-FSs (a) the $3 \mathrm{~d}$ view and (b) the Primary membership view. 


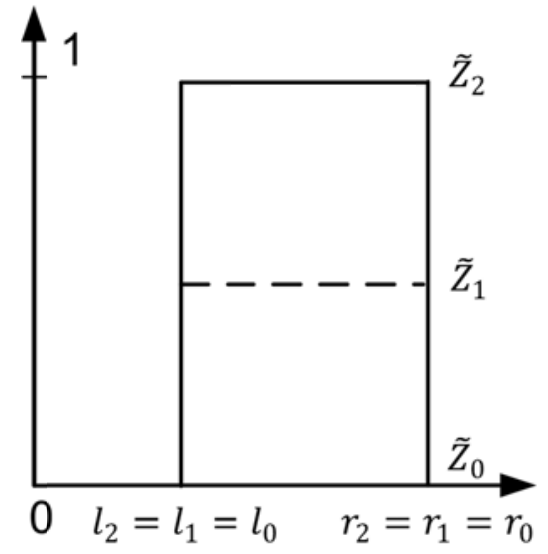

(a)

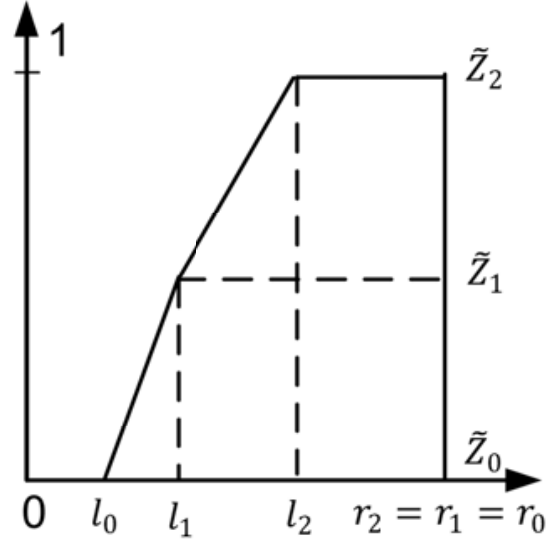

(b)

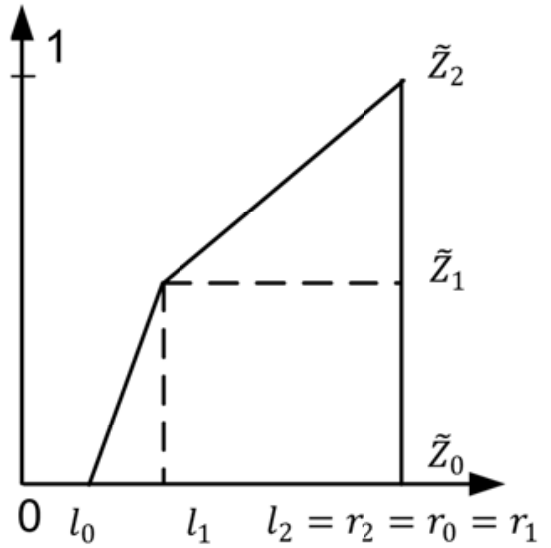

(c)

Fig. 4 Illustration of the SMFs (a) for $\gamma=0$ (b) for $0<\gamma<1$ (c) for $\gamma=1$.

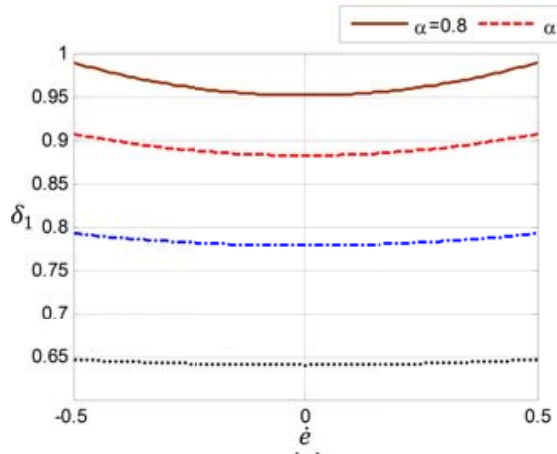

(a)

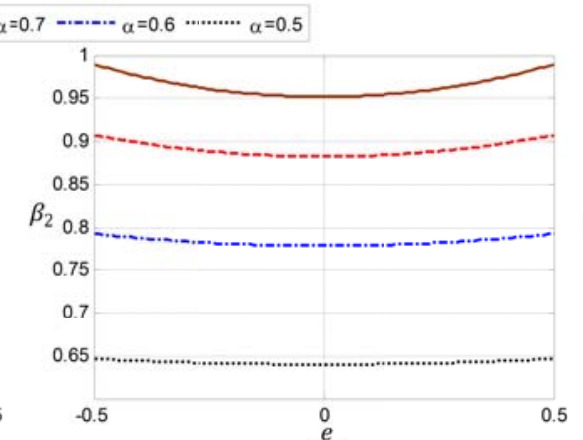

(b)

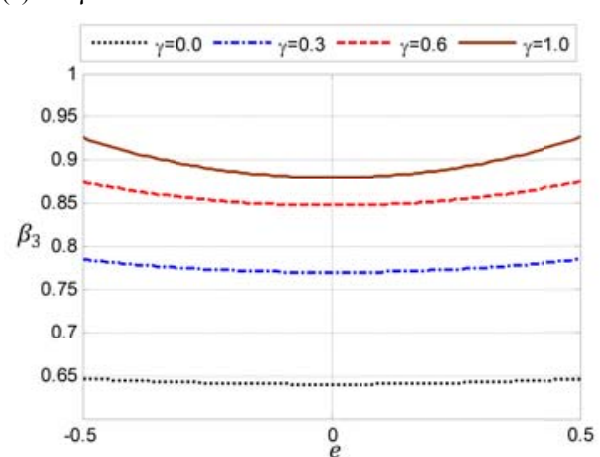

(c)

Fig. 5. Illustration of the relationship between (a) e and $\delta_{1}$ (b) e and $\beta_{2}$ (c) e and $\beta_{3}$

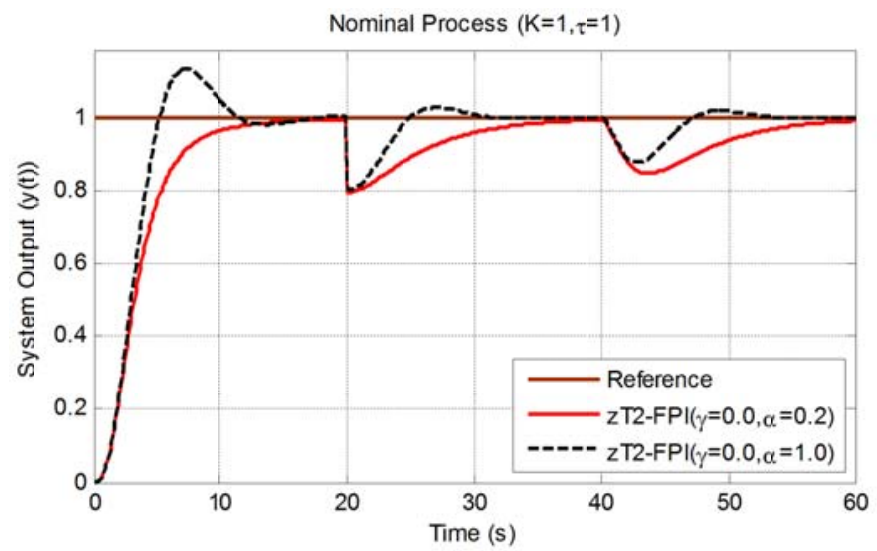

(a)

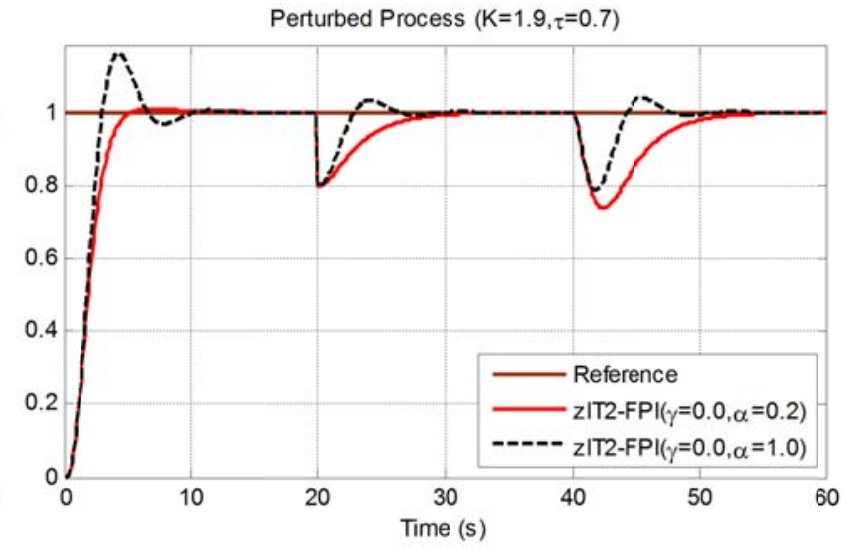

(b)

Fig. 6. Illustration of the control performance results for Case-2 the (a) nominal case (b) perturbed case 


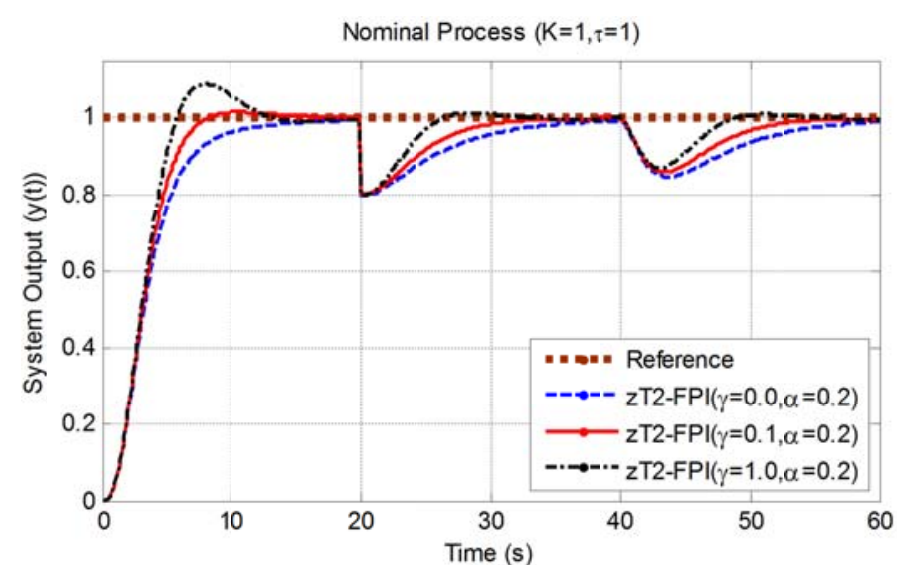

(a)

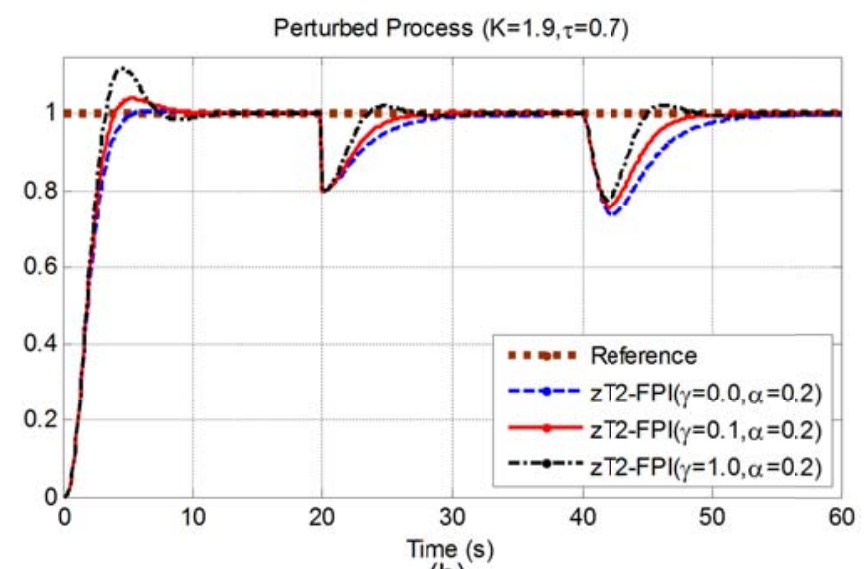

(b)

Fig. 7 Illustration of the control performance (a) for the nominal case (b) for the perturbed case.

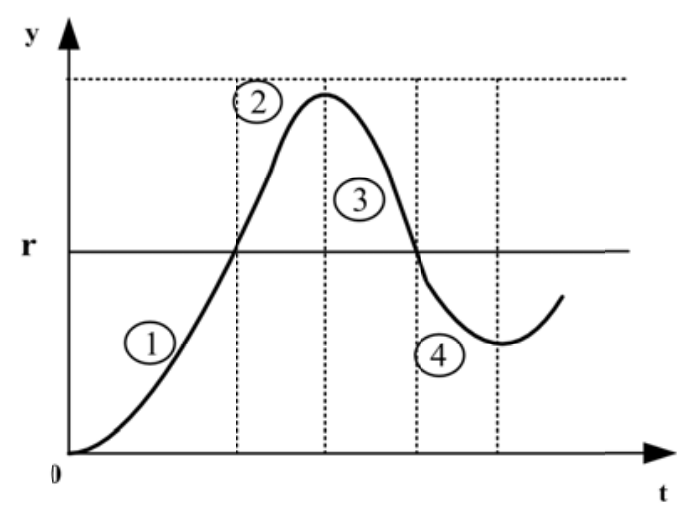

Fig.8 The partitioning of the step response into regions. 


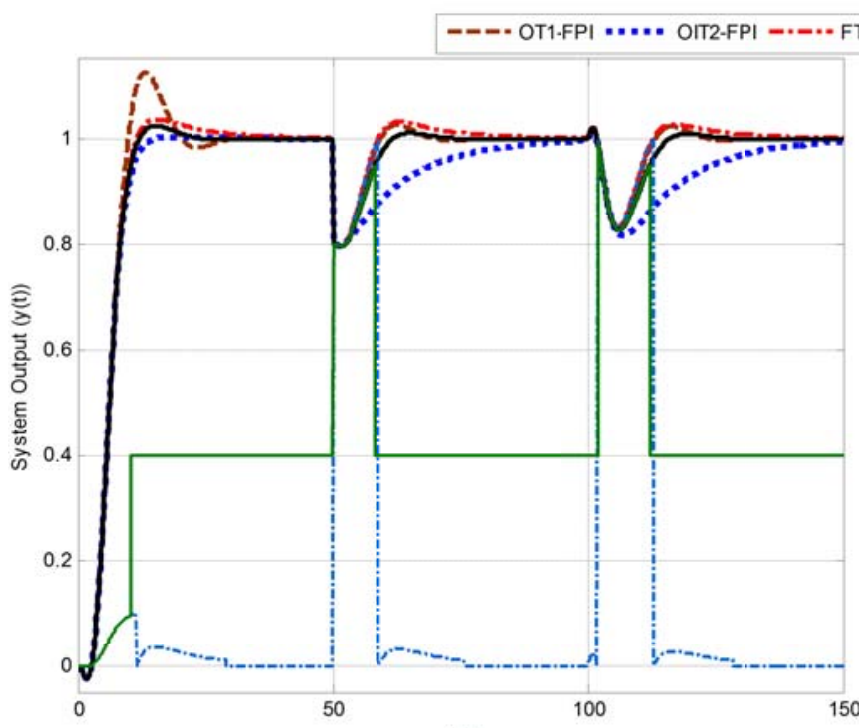

(a)

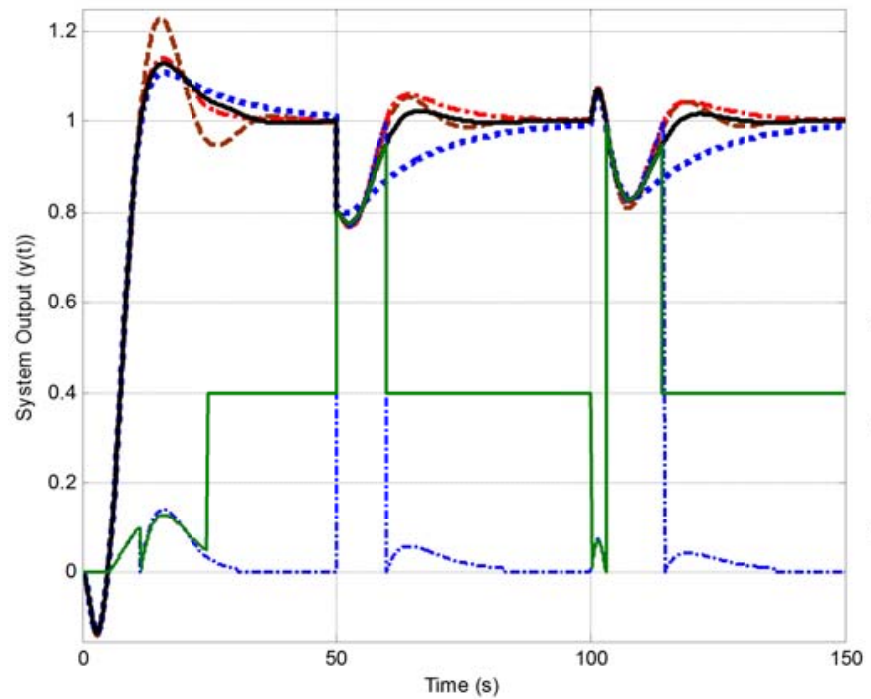

(c)

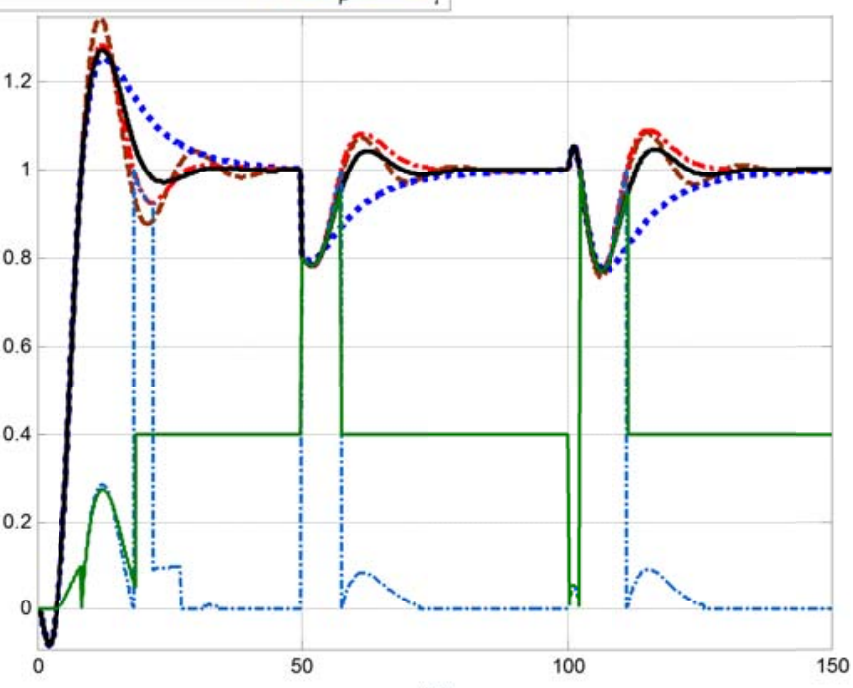

(b)

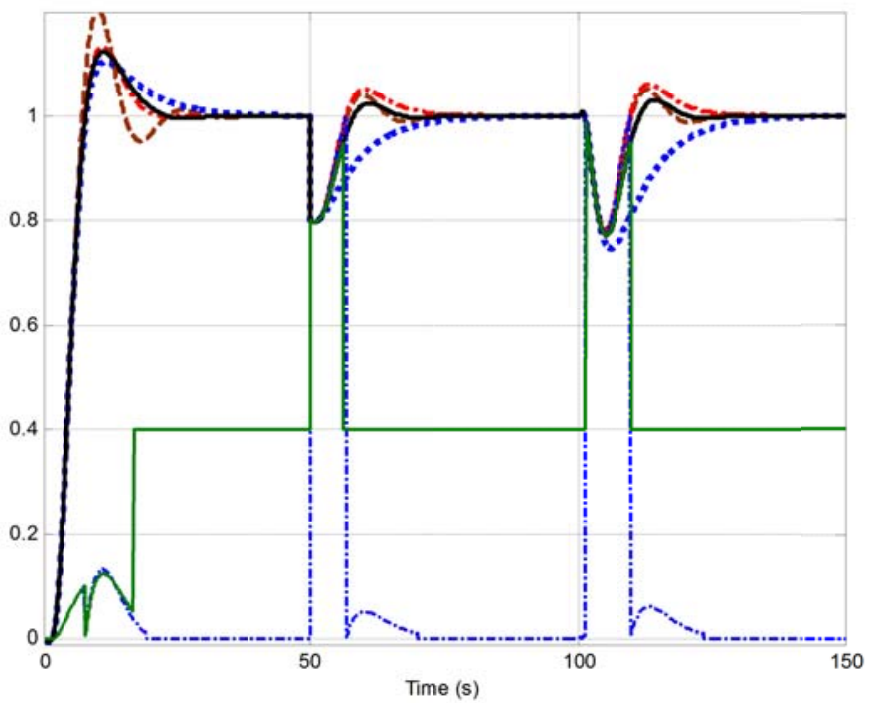

(d)

Fig. 9. Illustrations of the control performances for the System-I (a) Nominal System (b) Perturbed System-1(c) Perturbed System-2 (d) Perturbed System-3 


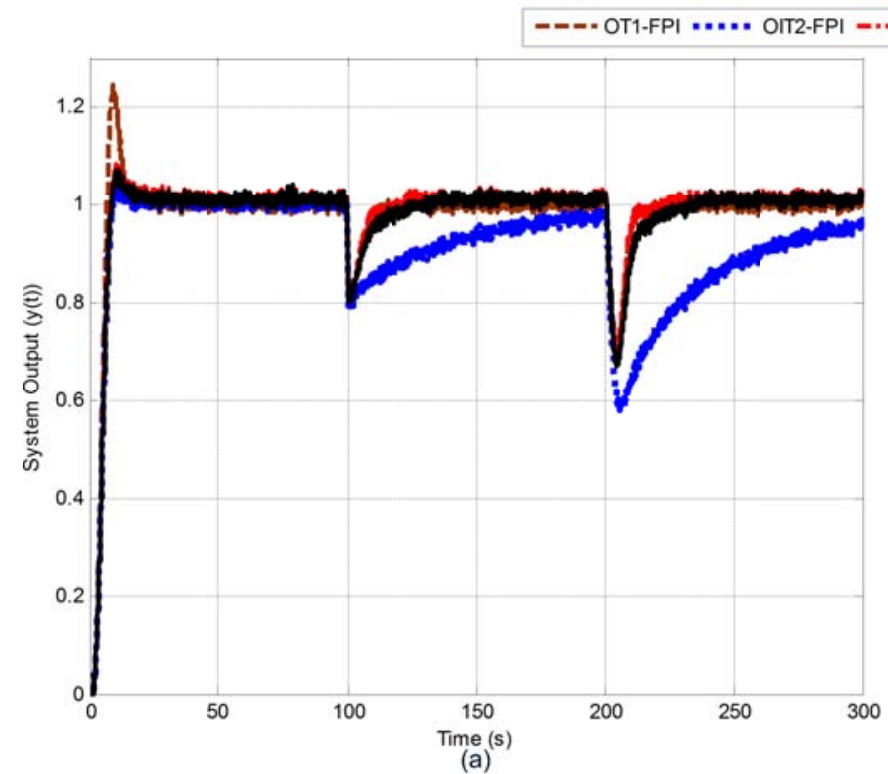

Fig. 10. Illustrations of the control performances for the System-II (a) Nominal System (b) Perturbed System

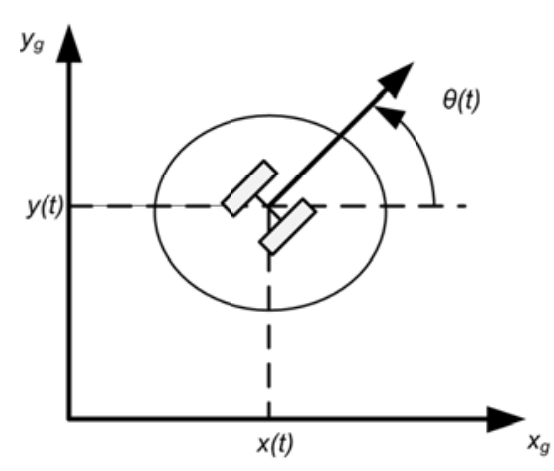

(a)

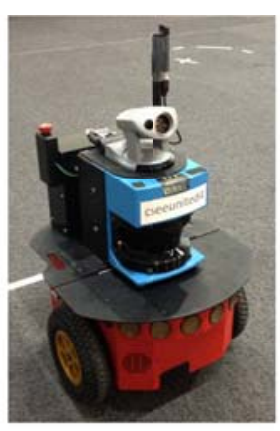

(b)

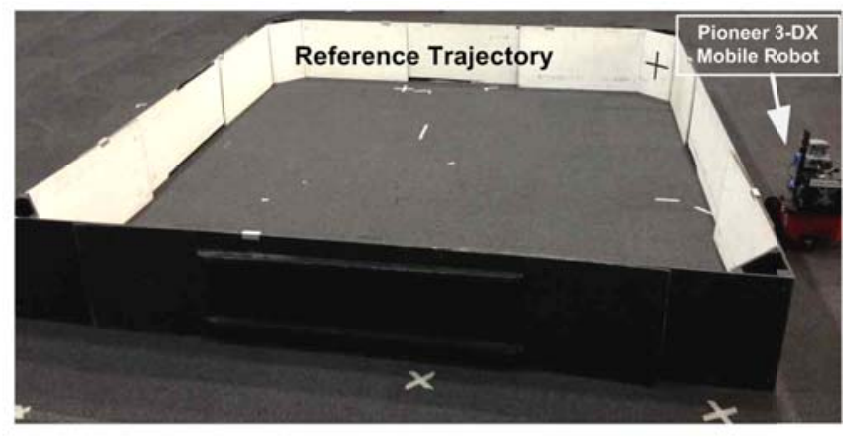

(c)

Fig. 11. Illustration of (a) the geometric description of the mobile robot (b) the general view of the Pioneer 3-DX mobile robot (c) the indoor environment of the Pioneer mobile robot

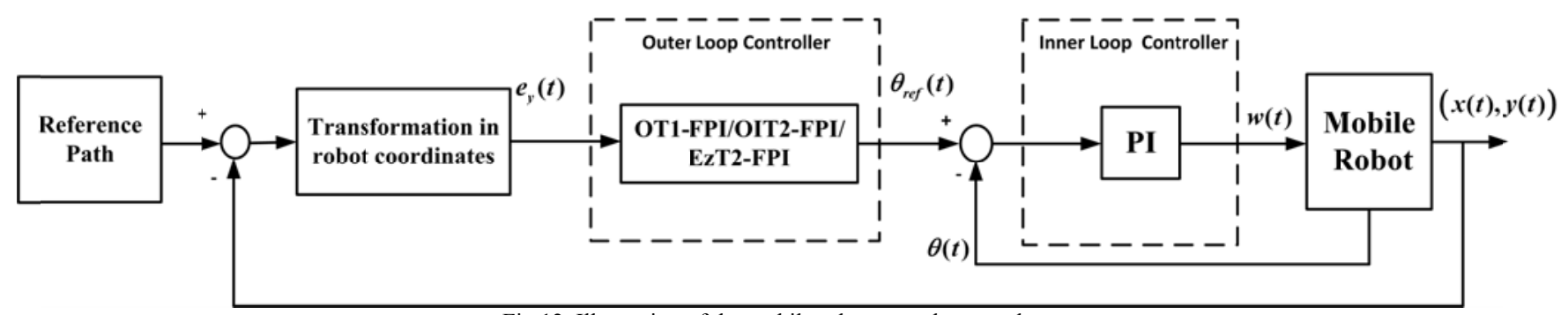

Fig.12. Illustration of the mobile robot cascade control structure. 


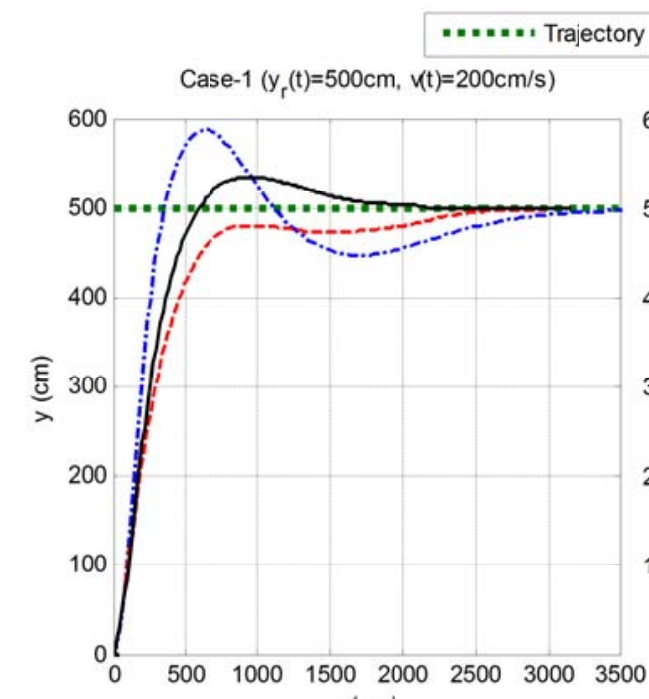

$x(\mathrm{~cm})$
$($ a)

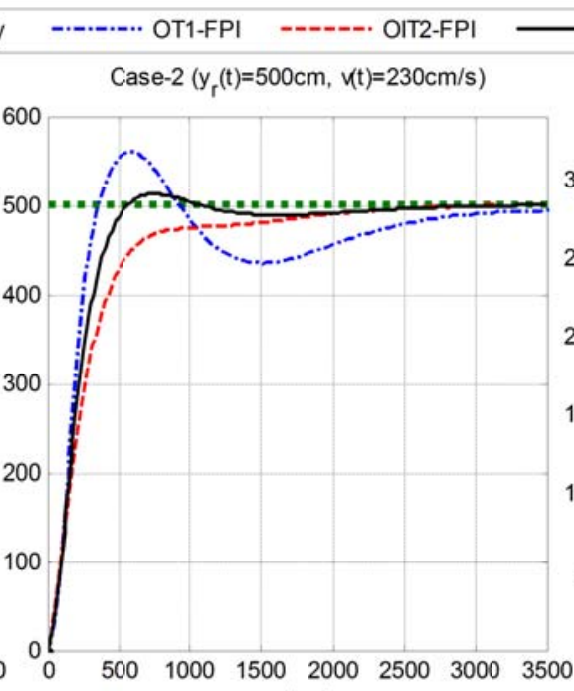

$x(\mathrm{~cm})$
EzT2-FPI

Case- $3\left(y_{r}(t)=300 \mathrm{~cm}, v(t)=200 \mathrm{~cm} / \mathrm{s}\right)$

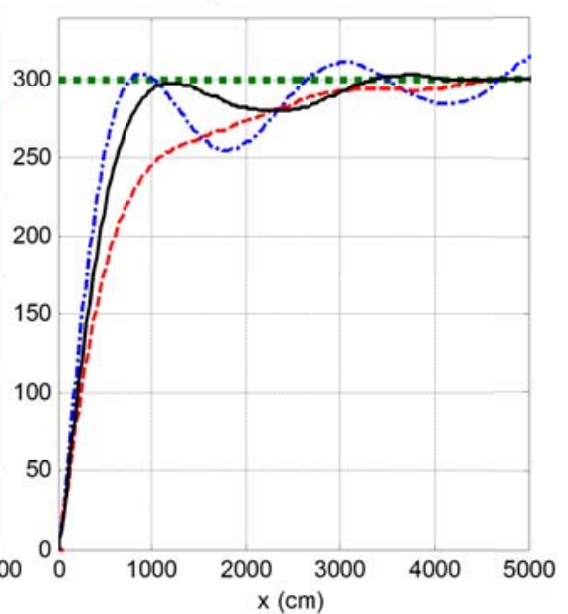

(c)

Fig. 13. Illustrations of the transient state performances for $y$-axis references for (a) Case-1 (b) Case-2 (c) Case-3

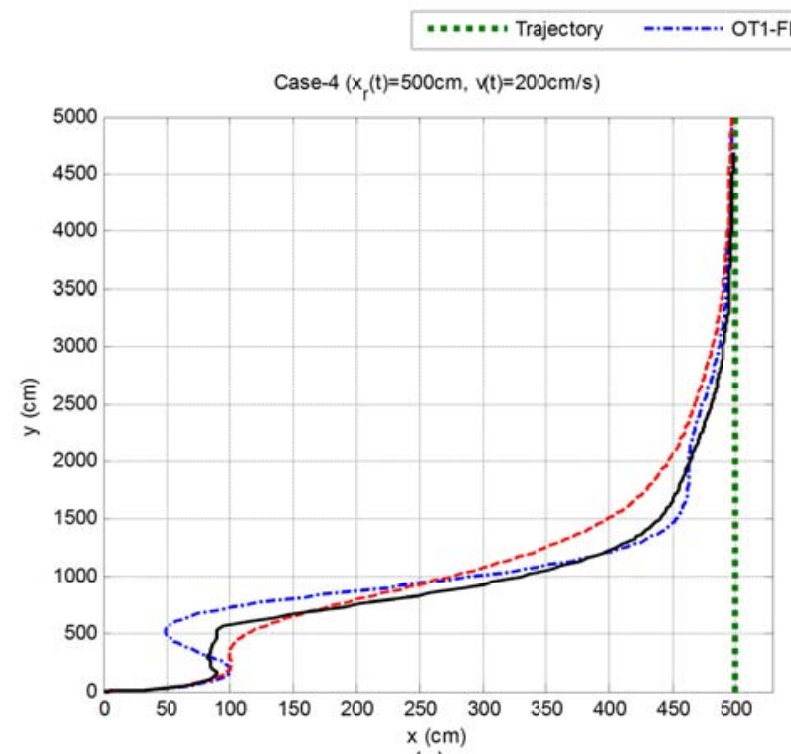

(a)
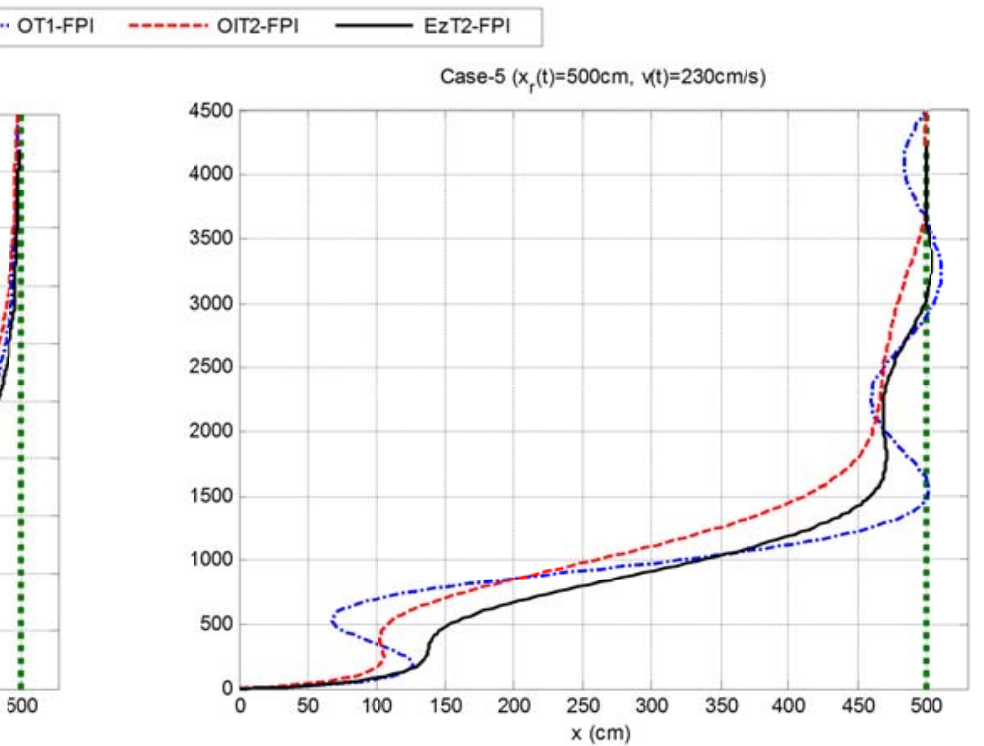

(b)

Fig. 14. Illustrations of the transient state performances for $\mathrm{x}$-axis references for (a) Case-4 (b) Case-5 


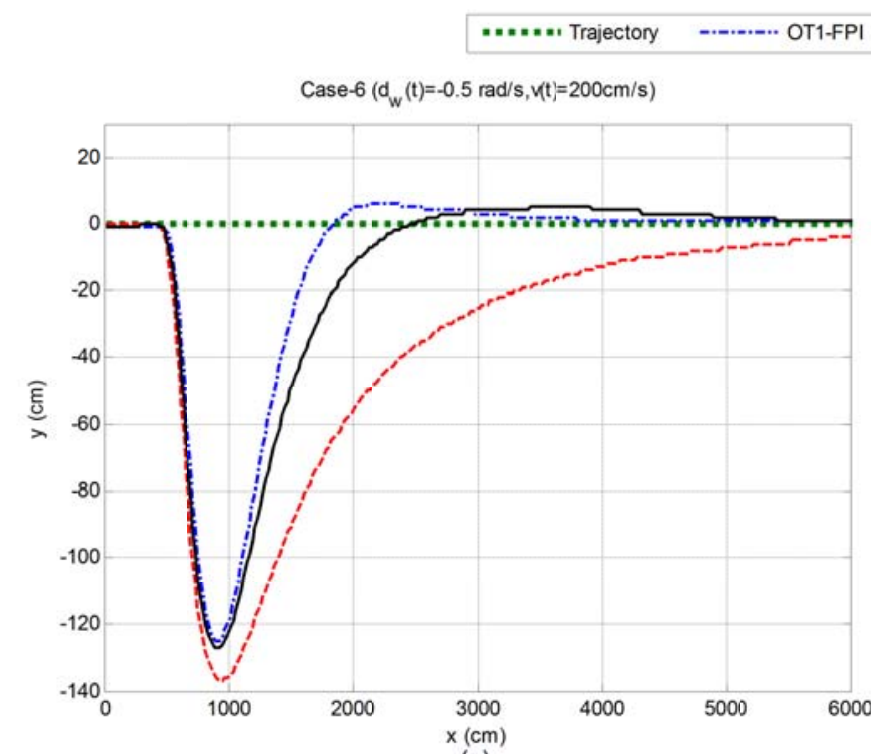

(a)

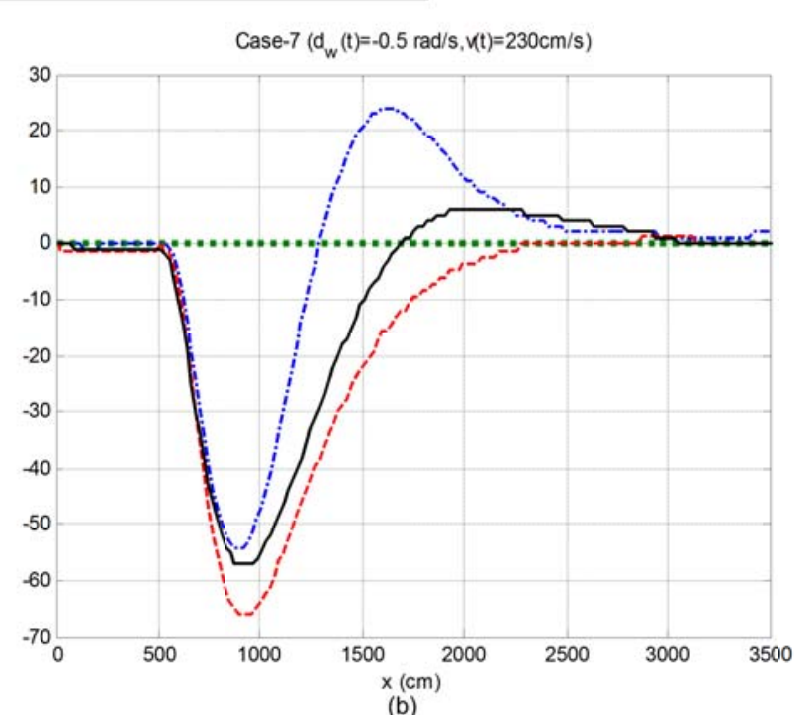

(b)

Fig. 15. Illustrations of the input disturbance rejection performances for (a) Case-6 (b) Case-7

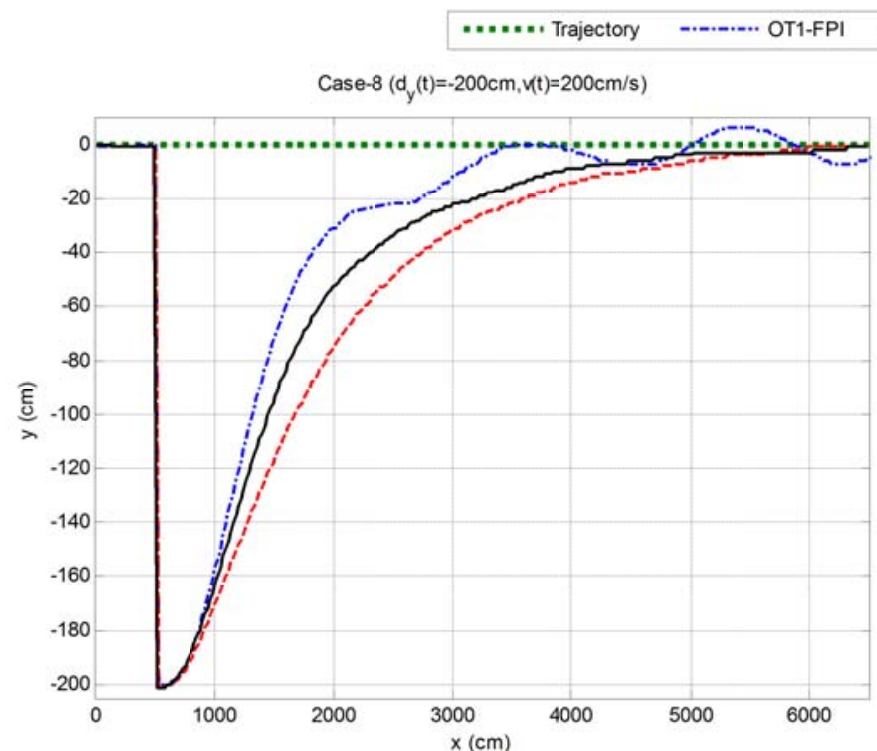

$\times(\mathrm{cm})$

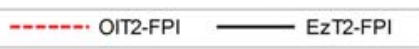

Case $-9\left(d_{y}(t)=-200 \mathrm{~cm}, v(t)=230 \mathrm{~cm} / \mathrm{s}\right)$

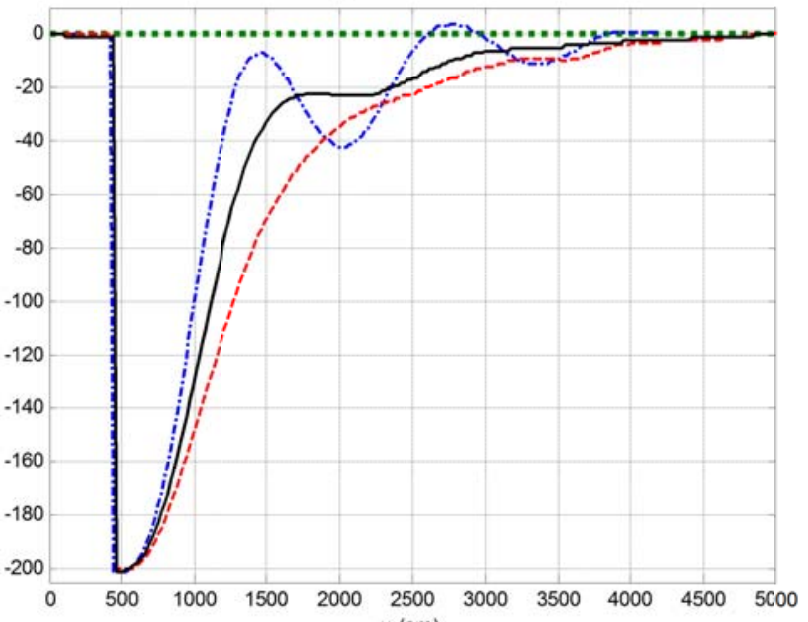

$\times(\mathrm{cm})$

Fig. 16. Illustrations of the output disturbance rejection performances for (a) Case-8 (b) Case-9 
TABle I. The PSEUdo-Code OF BB-BC BASEd FuZzy Controller Design Method

\begin{tabular}{ll}
\hline Step 1 & Define the parameters of the BB-BC optimization, i.e. the population size and the number of iterations. \\
Step 2 & Define the controller type (T1-PI/IT2-FPI) and the corresponding parameter set $\left(x_{T I-F P I} / x_{I T 2-F P I}\right)$ to be optimized. \\
Step 3 & Generate an initial population randomly for the parameter set of the controller (Initial Big Bang Phase). \\
Step 4 & $\begin{array}{l}\text { Simulate the closed loop control system for each population member. } \\
\text { Calculate the IAE performance values for the generated population members. }\end{array}$ \\
Step 5 & $\begin{array}{l}\text { Calculate the center of mass via Equation (85). } \\
\text { Choose the best fit individual or the center of mass as the point of Big Bang Phase (Big Crunch Phase). }\end{array}$ \\
Step 6 & Calculate new population members around the new point calculated in Step 6 via Equation (86). (Big Bang Phase). \\
Step 7 & Return to Step 4 until stopping criteria has been met. \\
\hline
\end{tabular}

TABLE II. THE PARAMETER SETS OF THE FUZZy PI CONTROLLERS

\begin{tabular}{|c|c|c|c|c|c|c|c|c|}
\hline & \multicolumn{4}{|c|}{ System-I } & \multicolumn{4}{|c|}{ System-II } \\
\hline & $\left(K_{P}, K_{I}\right)$ & $\left(\alpha_{\dot{e}}, \alpha_{e}\right)$ & $\left(\alpha_{\dot{e}_{0}}^{z_{1}}, \alpha_{e_{0}}^{z_{1}}\right)$ & $\left(\alpha_{\dot{e}}^{z_{1}}, \alpha_{e}^{z_{1}}\right)$ & $\left(K_{P}, K_{I}\right)$ & $\left(\alpha_{\dot{e}}, \alpha_{e}\right)$ & $\left(\alpha_{\dot{e}_{0}}^{z_{1}}, \alpha_{e_{0}}^{z_{1}}\right)$ & $\left(\alpha_{\dot{e}}^{z_{1}}, \alpha_{e}^{z_{1}}\right)$ \\
\hline OT1-FPI & $(0.20,0.21)$ & - & - & - & $(0.09,0.10)$ & - & - & - \\
\hline OIT2-FPI & $(0.20,0.21)$ & $(0.15,0.20)$ & - & - & $(0.09,0.10)$ & $(0.10,0.11)$ & - & - \\
\hline FT-zT2-FPI & $(0.20,0.21)$ & - & $(0.15,0.20)$ & - & $(0.09,0.10)$ & - & $(0.10,0.11)$ & - \\
\hline ST-zT2-FPI & $(0.20,0.21)$ & - & - & $(0.15,0.20)$ & $(0.09,0.10)$ & - & - & $(0.10,0.11)$ \\
\hline
\end{tabular}

TABLE III. TRANSIENT StATE PERFORMANCE COMPARISON OF THE FUZZY CONTROLLERS FOR SYSTEM-I

\begin{tabular}{|c|c|c|c|c|c|c|c|c|c|c|c|c|}
\hline \multirow[b]{3}{*}{ OT1-FPI } & \multicolumn{3}{|c|}{ Nominal System } & \multicolumn{3}{|c|}{ Perturbed System-1 } & \multicolumn{3}{|c|}{ Perturbed System-2 } & \multicolumn{3}{|c|}{ Perturbed System-3 } \\
\hline & OS & $\mathbf{T}_{\mathrm{s}}$ & IAE & OS & $\mathbf{T}_{\mathrm{s}}$ & IAE & OS & $\mathbf{T}_{\mathrm{s}}$ & IAE & OS & $\mathbf{T}_{\mathrm{s}}$ & IAE \\
\hline & $13 \%$ & $17.5 \mathrm{~s}$ & 7.15 & $34 \%$ & $25.2 \mathrm{~s}$ & 9.27 & $22 \%$ & $28.1 \mathrm{~s}$ & 10.41 & $21 \%$ & $18.1 \mathrm{~s}$ & 6.05 \\
\hline OIT2-FPI & $0 \%$ & $11.0 \mathrm{~s}$ & 6.59 & $25 \%$ & $28.6 \mathrm{~s}$ & 10.72 & $11 \%$ & $29.6 \mathrm{~s}$ & 10.34 & $10 \%$ & $19.2 \mathrm{~s}$ & 6.11 \\
\hline FT-zT2-FPI & $3 \%$ & $10.1 \mathrm{~s}$ & 6.91 & $27 \%$ & $25.1 \mathrm{~s}$ & 8.64 & $14 \%$ & $23.5 \mathrm{~s}$ & 9.91 & $13 \%$ & $16.2 \mathrm{~s}$ & 5.80 \\
\hline ST-zT2-FPI & $1 \%$ & $10.2 \mathrm{~s}$ & 6.60 & $26 \%$ & $18.6 \mathrm{~s}$ & 8.45 & $12 \%$ & $24.4 \mathrm{~s}$ & 9.85 & $11 \%$ & $16.8 \mathrm{~s}$ & 5.73 \\
\hline
\end{tabular}

TABLE IV. DistURBANCE REJeCTION PERFORMANCE COMPARISON OF THE FUZZY CONTROLLERS FOR SYSTEM-I

\begin{tabular}{|c|c|c|c|c|c|c|c|c|}
\hline \multirow{3}{*}{ OT1-FPI } & \multicolumn{2}{|c|}{ Nominal System } & \multicolumn{2}{|c|}{ Perturbed System-1 } & \multicolumn{2}{|c|}{ Perturbed System-2 } & \multicolumn{2}{|c|}{ Perturbed System-3 } \\
\hline & IAE $_{\text {dy }}$ & $\mathbf{I A E}_{\mathrm{du}}$ & $\mathbf{I A E}_{\mathrm{dy}}$ & $\mathbf{I A E}_{\mathrm{du}}$ & IAE $_{\mathrm{dy}}$ & $\mathbf{I A E}_{\mathrm{du}}$ & IAE $_{\mathrm{dy}}$ & IAE $_{\mathrm{du}}$ \\
\hline & $\begin{array}{l}1.38 \\
2.94\end{array}$ & $\begin{array}{l}1.28 \\
3.12\end{array}$ & $\begin{array}{l}1.81 \\
2.42\end{array}$ & $\begin{array}{l}2.19 \\
3.22\end{array}$ & 3.48 & $\begin{array}{l}1.80 \\
3.29\end{array}$ & $\begin{array}{l}1.15 \\
1.94\end{array}$ & $\begin{array}{l}1.40 \\
3.07\end{array}$ \\
\hline FT-zT2-FPI & 1.58 & 1.45 & 1.92 & 2.17 & 2.37 & 1.99 & 1.33 & 1.66 \\
\hline ST-zT2-FPI & 1.35 & 1.37 & 1.60 & 1.92 & 1.88 & 1.66 & 1.12 & 1.53 \\
\hline
\end{tabular}

TABLE V. PERFORMANCE COMPARISON OF THE FUZZY CONTROLLERS FOR SYSTEM-II

\begin{tabular}{|c|c|c|c|c|c|c|c|c|c|c|}
\hline & \multicolumn{5}{|c|}{ Nominal System Performance } & \multicolumn{5}{|c|}{ Perturbed System Performance } \\
\hline & \multicolumn{3}{|c|}{ Transient State } & \multicolumn{2}{|c|}{ Disturbance Rejection } & \multicolumn{3}{|c|}{ Transient State } & \multicolumn{2}{|c|}{ Disturbance Rejection } \\
\hline & OS & $\mathbf{T}_{\mathrm{s}}$ & IAE & $\mathbf{I A E}_{\text {dy }}$ & $\mathbf{I A E}_{\mathrm{du}}$ & OS & $\mathbf{T}_{\mathrm{s}}$ & IAE & IAE $_{\text {dy }}$ & IAE $_{\mathrm{du}}$ \\
\hline OT1-FPI & $23 \%$ & $15.5 \mathrm{~s}$ & 6.04 & 1.72 & 2.48 & $56 \%$ & $29.5 \mathrm{~s}$ & 9.91 & 1.76 & 5.12 \\
\hline OIT2-FPI & $6 \%$ & $10.5 \mathrm{~s}$ & 5.38 & 8.20 & 15.13 & $37 \%$ & $64.2 \mathrm{~s}$ & 15.88 & 4.75 & 13.96 \\
\hline FT-zT2-FPI & $8 \%$ & $10.8 \mathrm{~s}$ & 5.52 & 1.69 & 2.45 & $37 \%$ & $23.2 \mathrm{~s}$ & 9.59 & 1.69 & 5.22 \\
\hline ST-zT2-FPI & $7 \%$ & $10.7 \mathrm{~s}$ & 5.42 & 1.75 & 2.62 & $37 \%$ & $18.1 \mathrm{~s}$ & 8.16 & 1.24 & 4.88 \\
\hline
\end{tabular}

TABLE VI. PERFORMANCE COMPARISON OF THE FUZZY CONTROLLERS FOR THE Y-AXIS REFERENCE TRAJECTORIES

\begin{tabular}{cccccccccc}
\hline & \multicolumn{3}{c}{ Case-1 } & \multicolumn{3}{c}{ Case-2 } & \multicolumn{3}{c}{ Case-3 } \\
\cline { 2 - 10 } OT1-FPI & $\mathbf{T}_{\mathrm{s}}$ & $\mathbf{O S}$ & $\mathbf{I A E}_{\mathbf{y}}$ & $\mathbf{T}_{\mathrm{s}}$ & $\mathbf{O S}$ & $\mathbf{I A E}_{\mathbf{y}}$ & $\mathbf{T}_{\mathrm{s}}$ & $\mathbf{O S}$ & $\mathbf{I A E}_{\mathbf{y}}$ \\
OI & $17.6 \%$ & 188756 & $14.9 \mathrm{~s}$ & $12.2 \%$ & 191820 & Oscillating System Response \\
OIT2-FPI & $10.7 \mathrm{~s}$ & $0.0 \%$ & 175990 & $9.8 \mathrm{~s}$ & $0.0 \%$ & 159550 & $20.1 \mathrm{~s}$ & $0.0 \%$ & 201900 \\
EzT2-FPI & $8.0 \mathrm{~s}$ & $6.8 \%$ & 137984 & $4.7 \mathrm{~s}$ & $2.5 \%$ & 116710 & $15.6 \mathrm{~s}$ & $0.0 \%$ & 132927 \\
\hline
\end{tabular}

TABLE VII. PERFORMANCE COMPARISON OF THE FUZZY CONTROLLERS FOR THE X-AXIS REFERENCE TRAJECTORIES

\begin{tabular}{ccccccc}
\hline & \multicolumn{3}{c}{ Case-4 } & \multicolumn{3}{c}{ Case-5 } \\
\cline { 2 - 7 } OT1-FPI & $\mathbf{T}_{\mathbf{s}}$ & $\mathbf{O S}$ & $\mathbf{I A E}_{\mathbf{x}}$ & $\mathbf{T}_{\mathbf{s}}$ & $\mathbf{O S}$ & $\mathbf{I A E}_{\mathbf{x}}$ \\
OIT2-FPI & $16.2 \mathrm{~s}$ & $0.0 \%$ & 497930 & \multicolumn{2}{c}{ Oscillating System Response } \\
EzT2-FPI & $14.9 \mathrm{~s}$ & $0.0 \%$ & 509690 & $16.6 \mathrm{~s}$ & $0.0 \%$ & 511230 \\
\hline
\end{tabular}

TABLE VIII. DisTURBANCE REJECTION PERFORMANCE COMPARISON OF THE FUZZY CONTROLLERS

\begin{tabular}{ccccc}
\hline & $\begin{array}{c}\text { Input Disturbance Rejection } \\
\text { Case-6 }\end{array}$ & $\begin{array}{c}\text { Output Disturbance Rejection } \\
\text { Case-7 }\end{array}$ & $\begin{array}{c}\text { Case-8 } \\
\text { IAE }_{\text {dy }}\end{array}$ & Case-9 \\
& IAE $_{\text {dy }}$ \\
IAE $_{\text {du }}$ & IAE $_{\text {du }}$ & 311610 & $*$ & 166301 \\
OT1-FPI & 091031 & 310 & 298320 & 209960 \\
OIT2-FPI & 213180 & 381720 & 255700 & 159620 \\
\hline EzT2-FPI & 113460 & 271970 &
\end{tabular}

${ }^{*}$ Oscillating System Response 